\title{
Finite element solution of the Stokes problem with dominating Coriolis force
}

\author{
Ramon Codina*, Orlando Soto \\ International Center for Numerical Methods in Engineering, Universitat Politècnica de Catalunya, Gran Capità s/n, Edifici C1, \\ 08034 Barcelona, Spain
}

Received 4 January 1995; revised 27 February 1996

\begin{abstract}
The objective of this paper is to present a finite element formulation to solve the Stokes problem with Coriolis force. This force results in a skew-symmetric term in the weak formulation of the problem that deteriorates the stability of the standard Galerkin finite element method when the viscosity is small. We show that the stability is worsened due to the presence of the pressure gradient to enforce the incompressibility of the flow. The relevance of this effect depends on the relative importance of the viscous force and the Coriolis force, which is measured by the Ekman number. When it is small, oscillations occur using the Galerkin approach. To overcome them, we propose two different methods based on a consistent modification of the basic Galerkin formulation. Both methods eliminate the oscillations, keeping the accuracy of the formulation and enhancing its numerical stability.
\end{abstract}

\section{Introduction}

When the Navier-Stokes equations for an incompressible viscous fluid are written in a rotating frame of reference two new terms appear. One is the centrifugal force, which is independent of the velocity and the pressure and can be considered as a body force (or included in the pressure, since it can be written as the gradient of a scalar function). The other term is the Coriolis force, which can be expressed as $\omega \times \boldsymbol{u}$, where $\omega$ is twice the velocity of rotation of the frame of reference and $u$ is the velocity field referred to this rotating reference. Our interest in this work is focussed precisely on the effects that this term causes in the numerical solution of the flow equations and, in particular, in the Stokes problem. In the stationary case that we shall consider throughout, these equations are

$$
\begin{aligned}
& -\nu \Delta u+\nabla p+\omega \times u=f \\
& \nabla \cdot u=0,
\end{aligned}
$$

where $p$ is the kinematic pressure, $f$ is the vector of body forces (accounting also for the centrifugal force) and $\nu$ is the kinematic viscosity. The domain where the problem is to be solved (open, bounded and polyhedral) is denoted by $\Omega$.

Boundary conditions have to be appended to Eqs. (1). To simplify the exposition, we take the homogeneous Dirichlet prescription $\boldsymbol{u}=\mathbf{0}$ on the whole boundary $\partial \Omega$.

There are two main numerical difficulties associated with problem (1). The first of them is classical

\footnotetext{
* Corresponding author.
} 
and concerns the compatibility of the finite element spaces for the velocity and the pressure. It is well known (see e.g. [1]) that they have to satisfy the so-called Babuška-Brezzi stability condition. There is also the possibility of using the same interpolation for both the velocity and the pressure by modifying the standard Galerkin variational form (see [2-4] for examples of such methods). We shall describe one of these possibilities later on, namely, the Galerkin/least-squares method. Its extension to problem (1) is precisely one of the methods that allow to solve the second numerical difficulty described next.

The other difficulty encountered when one tries to solve problem (1) with very small values of the viscosity is the presence of spurious oscillations when the standard Galerkin finite element formulation is used. We shall show that they are due to the pressure $p$, which may be understood as a Lagrange multiplier to enforce the incompressibility of the flow dictated by Eq. (1b). If this incompressibility were not imposed, small viscosities could lead to local oscillations, only in the neighborhood of the boundary layers, but not to global ones. This phenomenon is well known and appears in problems with dominant absorption terms, that is, with terms proportional to the unknown function. In this case it is not possible to obtain a global stability estimate in the $H^{1}$ norm, although it is in the $L^{2}$ one, thus explaining why these local oscillations may exist but can not deteriorate the solution globally. However, for problem (1) it is not possible to obtain the aforementioned estimates (at least in a straightforward manner) due to the presence of the pressure. Therefore, not only local boundary layer oscillations may appear, but also global ones may be expected. The situation is, in a sense, similar to what happens in the linear convection-diffusion equation when the diffusive term is very small compared to the convective one-the standard Galerkin method has a global lack of stability. In our case the study is complicated by the fact that the phenomenon is genuinely multi-dimensional.

The dimensionless number that allows to quantify the relative importance of the viscous and the

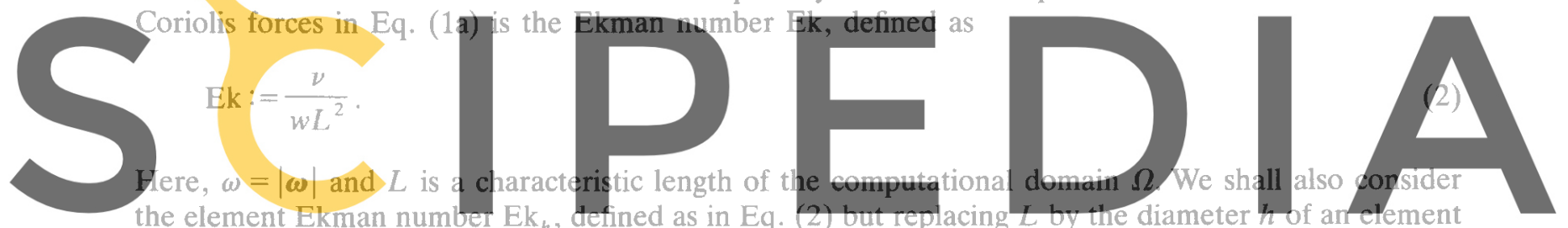
under consideration

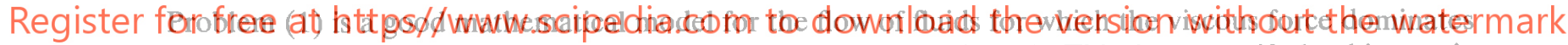
the convective one and therefore inertial effects can be neglected. This happens if the kinematic viscosity is large and thus we should consider the angular velocity very large in order to have a physically meaningful situation with small Ekman number and negligible inertia. However, and only to fix ideas, we shall consider that small Ekman number flows correspond to cases in which the viscosity is small. The model problem studied in this paper and the numerical techniques developed to solve it must be considered only as a first step towards the solution of the complete Navier-Stokes equations written in a rotating frame of reference.

Our purpose in this work is to develop a finite element formulation free of spurious oscillations even in the case in which Ek is very small, that is, when the Coriolis force dominates the viscous one. This will be accomplished by adding to the original Galerkin formulation stabilizing terms that shall be described in Sections 3 and 4, after presenting in the following section the standard Galerkin formulation of the problem and discussing the origin of its misbehavior. In Section 5 some numerical experiments will be presented, showing the type of oscillations that can be found using the Galerkin approach and how the stabilizing techniques proposed in this paper allow to remove them.

\section{Galerkin finite element approximation}

\subsection{Statement of the problem}

Let $n_{\mathrm{sd}}=2$ or 3 be the number of space dimensions and consider the following function spaces 


$$
\begin{aligned}
& V=\left(H_{0}^{1}(\Omega)\right)^{n_{\mathrm{sd}}} \\
& Q=\left\{q \in L^{2}(\Omega) \mid \int_{\Omega} q \mathrm{~d} \Omega=0\right\}
\end{aligned}
$$

and the forms

$$
\begin{aligned}
& a(\boldsymbol{u}, \boldsymbol{v})=\nu \int_{\Omega} \nabla \boldsymbol{u}: \nabla v \mathrm{~d} \Omega \\
& b(q, \boldsymbol{v})=\int_{\Omega} q \nabla \cdot \boldsymbol{v} \mathrm{d} \Omega \\
& c(\boldsymbol{u}, \boldsymbol{v})=\int_{\Omega}(\boldsymbol{\omega} \times \boldsymbol{u}) \cdot \boldsymbol{v} \mathrm{d} \Omega \\
& l(\boldsymbol{v})=\int_{\Omega} f \cdot \boldsymbol{v} \mathrm{d} \Omega
\end{aligned}
$$

with $u, v \in V$ and $q \in Q$. Having introduced this notation, the weak form of problem (1) consists in finding a velocity $u \in V$ and a pressure $p \in Q$ such that

$$
\begin{array}{ll}
a(u, v)-b(p, v)+c(u, v)=l(v) & \forall v \in V \\
b(q, u)=0 & \forall q \in Q
\end{array}
$$

Existence and uniqueness of solution for this problem can be proved exactly as for the classical Stokes problem without the Coriolis force. Observe that the bilinear form $c(u, v)$ is continuous and skew-symmetric and thus $a(u, v)$ this space.

The finitc element approximation of problem (5) that we consider is obtaingd simply and $Q$ by finite element subspaces $V_{h} \subset V$ and $Q_{h} \subset Q$ (here and bolow, we introduce tefer to the discrete finite element problem). This is the standard conforming Galerkin approximation of the problem.

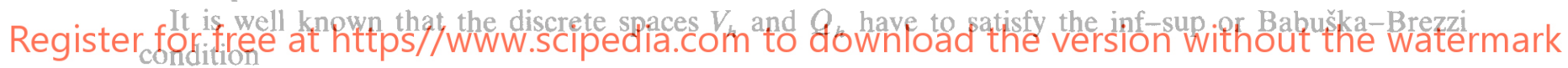

$$
\sup _{\boldsymbol{v}_{h}} \frac{b\left(q_{h}, v_{h}\right)}{\left\|\boldsymbol{v}_{h}\right\|_{1}} \geqslant K_{b}\left\|q_{h}\right\|_{0} \quad \forall q_{h} \in Q_{h},
$$

where the supremum is taken over all the $v_{h} \in V_{h} \backslash\{0\}, K_{b}$ is a positive constant and $\|\cdot\|_{0}$ and $\|\cdot\|_{1}$ are the standard $L^{2}$ and $H^{1}$ norms, respectively.

In the method to be described in Section 4 we shall assume that condition (6) holds, although it will not be necessary in the method presented in Section 3, since it allows to circumvent it. Several combinations of velocity and pressure interpolations that satisfy condition (6) can be found, e.g. in $[5,6]$. Some of them will be described in Section 5 and used in the numerical example.

\subsection{Stability properties of the Galerkin method}

We are interested now in studying the stability properties of the discrete finite element version of problem (5). First, let us introduce the bilinear form $\mathscr{A}$ acting on $\left(V_{h} \times Q_{h}\right) \times\left(V_{h} \times Q_{h}\right)$ and defined as

$$
\mathscr{A}\left(\boldsymbol{u}_{h}, p_{h} ; \boldsymbol{v}_{h}, q_{h}\right):=a\left(\boldsymbol{u}_{h}, \boldsymbol{v}_{h}\right)-b\left(p_{h}, \boldsymbol{v}_{h}\right)+c\left(\boldsymbol{u}_{h}, \boldsymbol{v}_{h}\right)+b\left(q_{h}, \boldsymbol{u}_{h}\right)
$$

and the linear form $\mathscr{L}$ defined on $V_{h} \times Q_{h}$ as

$$
\mathscr{L}\left(\boldsymbol{v}_{h}, q_{h}\right):=l\left(\boldsymbol{v}_{h}\right) .
$$

The discrete counterpart of problem (5) can now be written as: find a pair $\left(u_{h}, p_{h}\right) \in V_{h} \times Q_{h}$ such that 


$$
\mathscr{A}\left(\boldsymbol{u}_{h}, p_{h} ; \boldsymbol{v}_{h}, q_{h}\right)=\mathscr{L}\left(\boldsymbol{v}_{h}, q_{h}\right)
$$

for all $\left(v_{h}, q_{h}\right) \in V_{h} \times Q_{h}$.

The numerical stability of the problem is provided by the coercivity of the bilinear form $\mathscr{A}$ in the space $V_{h}$ and by condition (6). Let $\alpha \geqslant 0$ be a given scalar. It may be readily checked that

$$
\mathscr{A}\left(\boldsymbol{u}_{h}, p_{h} ; \boldsymbol{u}_{h}+\alpha\left(\boldsymbol{\omega} \times \boldsymbol{u}_{h}\right), p_{h}\right)=\nu\left\|\nabla \boldsymbol{u}_{h}\right\|_{0}^{2}+\alpha\left\|\boldsymbol{\omega} \times \boldsymbol{u}_{h}\right\|_{0}^{2}+\alpha \int_{\Omega} p_{h} \boldsymbol{\omega} \cdot\left(\nabla \times \boldsymbol{u}_{h}\right) \mathrm{d} \Omega,
$$

where we have made use of the relations

$$
\begin{aligned}
& \boldsymbol{u}_{h} \cdot\left(\boldsymbol{\omega} \times \boldsymbol{u}_{h}\right)=0 \\
& \nabla \boldsymbol{u}_{h}: \nabla\left(\boldsymbol{\omega} \times \boldsymbol{u}_{h}\right)=0 \\
& \nabla \cdot\left(\boldsymbol{\omega} \times \boldsymbol{u}_{h}\right)=-\boldsymbol{\omega} \cdot\left(\nabla \times \boldsymbol{u}_{h}\right) .
\end{aligned}
$$

From Eq. (10) it is observed that for $\alpha=0$ we have control over $\left\|\nabla u_{h}\right\|_{0}, u_{h}$ being the solution of problem (9). By invoking the Poincaré-Friedrics inequality we could obtain an a priori bound for $\left\|u_{h}\right\|_{1}$. However, these estimates are multiplied by the inverse of the kinematic viscosity $\nu$. If it is very small, they are useless from the numerical standpoint and the Coriolis force $\omega \times u_{h}$ will be completely out of control. Observe that if the problem is written in dimensionless form $\nu$ may be replaced by the Ekman number Ek defined in Eq. (2).

If we take $\alpha>0$ in Eq. (10) it is seen that the possible control that we could have over $\omega \times u_{h}$ may be destroyed by the last term in this equation. It is clear that this term would not appear if we do not

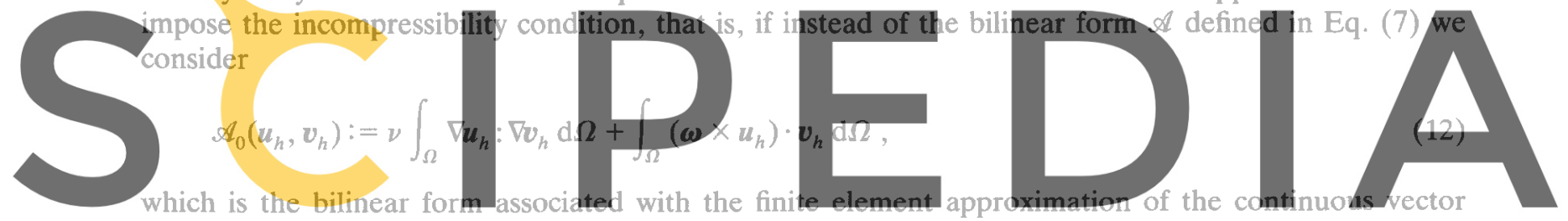

equation

Register for free at bttas $\$$ /Nwww.scipedia.com to download the version without the watermark

with homogeneous Dirichlet boundary conditions. Therefore, the possible lack of stability due to the term $\boldsymbol{\omega} \times \boldsymbol{u}_{h}$ is a problem originated exclusively by the presence of the pressure $p_{h}$ associated with the (weakly imposed) incompressibility of the flow. The solution of Eq. (13) using finite elements may exhibit only local boundary layer oscillations. They will be further analysed in the following subsection.

Numerical experiments indicate that the above-mentioned lack of stability in problem (9) in fact exists. Global oscillations occur when the element Ekman number is very small.

-From Eq. (10) it is observed that if the angular velocity $\omega$ is orthogonal to the vorticity $\nabla \times u_{h}$ then it is possible to bound $\left\|u_{h}\right\|_{0}$ using the standard Galerkin method. This must be kept in mind since the modification of the original formulation described Section 4 disappears when this orthogonality holds. Note also that this situation can only be found in three-dimensional problems (for non-zero vorticities).

\subsection{Truncation error for a $1 D$ model problem}

This subsection is intended to get more insight in the behavior of the numerical solution of Eq. (13) with Dirichlet conditions $\boldsymbol{a}=\mathbf{0}$ on $\partial \Omega$. For that, let us consider the following simple one-dimensional problem:

$$
\begin{array}{ll}
-\nu \frac{\mathrm{d}^{2} u}{\mathrm{~d} x^{2}}-\omega v=f_{1}, & 0<x<1 \\
-\nu \frac{\mathrm{d}^{2} v}{\mathrm{~d} x^{2}}+\omega u=f_{2}, & 0<x<1
\end{array}
$$




$$
u(0)=u(1)=v(0)=v(1)=0,
$$

where $u(x)$ and $v(x)$ are the unknown functions and $f_{1}$ and $f_{2}$ are constants. Except for the boundary conditions (that can be easily generalized), this models for example the so-called Ekman problem (see e.g. [7]). As mentioned previously, no global oscillations have to be expected when the standard Galerkin approximation is employed. Only localized overshoots and undershoots may appear near the boundarics for very small values of the viscosity $\nu$, in which case boundary layers are created.

If the interval $[0,1]$ is discretized using a uniform partition of linear finite elements of length $h$, the standard Galerkin approximation applied to problem (14) leads to the following set of difference equations:

$$
\begin{aligned}
& -\hat{u}_{i-1}+2 \hat{u}_{i}-\hat{u}_{i+1}-\frac{w h^{2}}{6 \nu}\left(\hat{v}_{i-1}+4 \hat{v}_{i}+\hat{v}_{i+1}\right)=\frac{h^{2}}{\nu} f_{1} \\
& -\hat{v}_{i-1}+2 \hat{v}_{i}-\hat{v}_{i+1}+\frac{w h^{2}}{6 v}\left(\hat{u}_{i-1}+4 \hat{u}_{i}-\hat{u}_{i+1}\right)=\frac{h^{2}}{v} f_{2},
\end{aligned}
$$

where $\hat{u}_{j}$ and $\hat{v}_{j}$ are the nodal values of the approximated unknown functions at the $j$ th node of the mesh and $i$ above stands for an interior node, the abcissa of which is denoted by $x_{i}$ in the following. Let us concentrate on the truncation error for Eq. (15a). If $u$ and $v$ are the solution of problem (14), we use the abbreviations

$$
u_{i}^{(n)}:=\left.\frac{\mathrm{d}^{n} u}{\mathrm{~d} x^{n}}\right|_{x=x_{i}} \quad \text { and } \quad u_{i}:=u\left(x_{i}\right)
$$
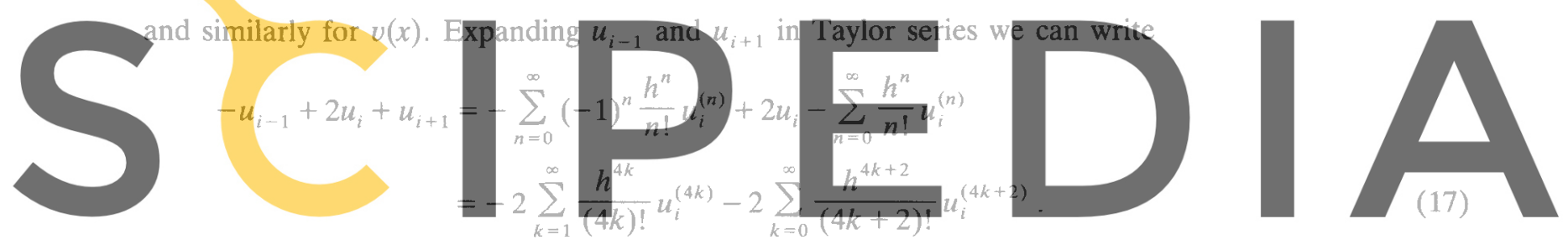

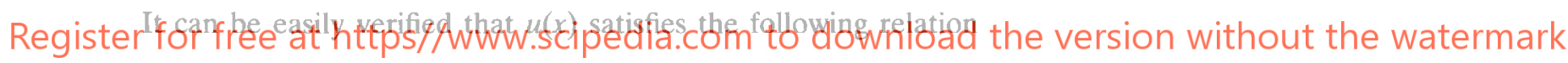

$$
\frac{\mathrm{d}^{4 k} u}{\mathbf{d} \boldsymbol{x}^{4 k}}=(-1)^{k} \frac{\omega^{2 k}}{\boldsymbol{\nu}^{2 k}}\left(u-\frac{f_{2}}{\omega}\right), \quad k=1,2,3, \ldots
$$

Using this in Eq. (17) it is found that

$$
\begin{aligned}
-u_{i-1}+2 u_{i}+u_{i+1}= & 2\left[\frac{f_{2}}{\omega}-u_{i}\right]\left[\sum_{k=0}^{\infty}(-1)^{k}\left(\sqrt{\frac{\omega}{\nu}} h\right)^{4 k} \frac{1}{(4 k) !}-1\right] \\
& -2 u_{i}^{(2)} \frac{\nu}{\omega} \sum_{k=0}^{\infty}(-1)^{k}\left(\sqrt{\frac{\omega}{\nu}} h\right)^{4 k+2} \frac{1}{(4 k+2) !} .
\end{aligned}
$$

The series appearing in this expression can be summed up and expressed in terms of simple functions. This yields

$$
\begin{aligned}
-u_{i-1}+2 u_{i}+u_{i+1}= & 2\left[\frac{f_{2}}{\omega}-u_{i}\right]\left[\cos \left(\sqrt{\frac{\omega}{2 \nu}} h\right) \cosh \left(\sqrt{\frac{\omega}{2 \nu}} h\right)-1\right] \\
& -2 u_{i}^{(2)} \frac{\nu}{\omega}\left[\sin \left(\sqrt{\frac{\omega}{2 \nu}} h\right) \sinh \left(\sqrt{\frac{\omega}{2 \nu}} h\right)\right]
\end{aligned}
$$

and a similar expression can be obtained replacing $u$ by $v$ and $\omega$ by $-\omega$. Introducing the dimensionless parameter

$$
\lambda:=\sqrt{\frac{\omega h^{2}}{2 \nu}}
$$

and making use of Eq. (20) (and the analogous for $v$ ) we obtain that 


$$
-u_{i-1}+2 u_{i}+u_{i+1}-\frac{\omega h^{2}}{6 \nu}\left(v_{i-1}+4 v_{i}+v_{i+1}\right)=\frac{h^{2}}{\nu}\left(-v u_{i}^{(2)}-\omega v_{i}\right)+F_{u, i},
$$

where $E_{u, i}$ is the truncation error for the first equation of system (15) multiplied by $h^{2} / \nu$ at the $i$ th node. It is given by

$$
\begin{aligned}
E_{u, i}= & -2 \frac{\nu}{\omega} v_{i}^{(2)}\left[\cos (\lambda) \cosh (\lambda)-\frac{\lambda^{2}}{3} \sin (\lambda) \sinh (\lambda)-1\right] \\
& -2 \frac{\nu}{\omega} u_{i}^{(2)}\left[\sin (\lambda) \sinh (\lambda)-\frac{\lambda^{2}}{3} \cos (\lambda) \cosh (\lambda)+2\right] .
\end{aligned}
$$

The value of the truncation error at each point is now found by replacing in Eq. (23) the second derivatives of $u$ and $v$ by their exact expressions. After doing this, one finds that

$$
\begin{aligned}
E_{u, i}= & 2\left[C_{3} \exp \left(\alpha x_{i}\right) \sin \left(\alpha x_{i}\right)-C_{2} \exp \left(-\alpha x_{i}\right) \cos \left(\alpha x_{i}\right)\right] \\
& \times\left[\sin (\lambda) \sinh (\lambda)-\frac{\lambda^{2}}{3}(\cos (\lambda) \cosh (\lambda)+2)\right] \\
& +2\left[C_{4} \exp \left(\alpha x_{i}\right) \sin \left(\alpha x_{i}\right)-C_{1} \exp \left(-\alpha x_{i}\right) \cos \left(\alpha x_{i}\right)\right] \\
& \times\left[\cos (\lambda) \cosh (\lambda)-\frac{\lambda^{2}}{3} \sin (\lambda) \sinh (\lambda)-1\right],
\end{aligned}
$$

where $\alpha:=\lambda / h$, and the constants $C_{i}, j=1,2,3,4$, are given by
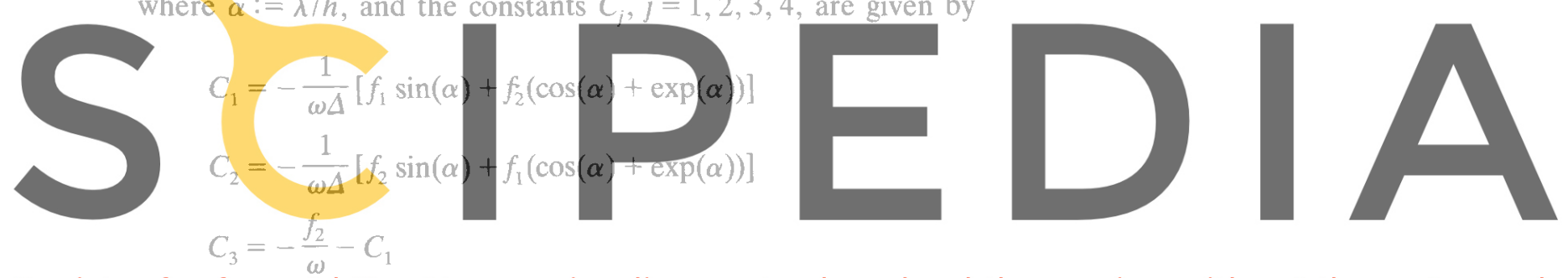

Register for free $f_{1}$ at https//www.scipedia.com to download the version without the watermark

$$
\begin{aligned}
& C_{4}=\frac{1}{\omega}-C_{2} \\
& \Delta=\exp (-\alpha)+2 \cos (\alpha)+\exp (\alpha) .
\end{aligned}
$$

We are interested now in studying the behavior of $E_{u, i}$ in the limit cases $\omega \rightarrow 0$ and $\nu \rightarrow 0$. Let us consider the case of small $\lambda$, either because $\omega$ is very small or because $h \rightarrow 0$. From the expression of the constants $C_{j}, j=1,2,3,4$, in (25) it is easy to see that they remain bounded as $\lambda \rightarrow 0$. Moreover, since the bracketed functions of $\lambda$ in Eq. (24) are of order $\lambda^{4}$, the truncation error $\nu E_{u, i} / h^{2}$ will be of order $\lambda^{2}$ for all the nodal points. This is what one could expect from the discretization of the equations that we have used.

What is more important for us is the case $\nu \rightarrow 0$ (for a fixed $\omega$ ). From the expressions (25) it is found that $C_{1} \rightarrow-f_{2} / \omega$ and $C_{2} \rightarrow f_{1} / \omega$ as $\alpha \rightarrow \infty$, whereas $C_{3}$ and $C_{4}$ behave like $\exp (-\alpha)$. Therefore, up to constant factors (positive or negative), $E_{u, i}$ behaves like

$$
\left[\exp (-\alpha) \exp \left(\alpha x_{i}\right) \exp (\alpha h)+\exp \left(-\alpha x_{i}\right) \exp (\alpha h)\right]\left[1+\alpha^{2} h^{2}\right]
$$

From this it is observed that the truncation error will tend to zero in the interior of the domain, and will tend to infinity at the nodes next to the boundary. This reflects the typical boundary layer oscillations found also in scalar equations with a dominating absorption term [8-10]. It is important to observe that these oscillations are localized, and that they can not propagate towards the interior of the computational domain. Thus, at least for the linear equations that we are considering and for problems with smooth solutions, they are only a minor problem from the practical point of view.

In Fig. 1 we have plotted the absolute value of $E_{u, i}$ for different values of the viscosity. In this case, we have discretized the interval $[0,1]$ using 20 linear elements and we have taken $f_{1}=f_{2}=1, \omega=1$. 


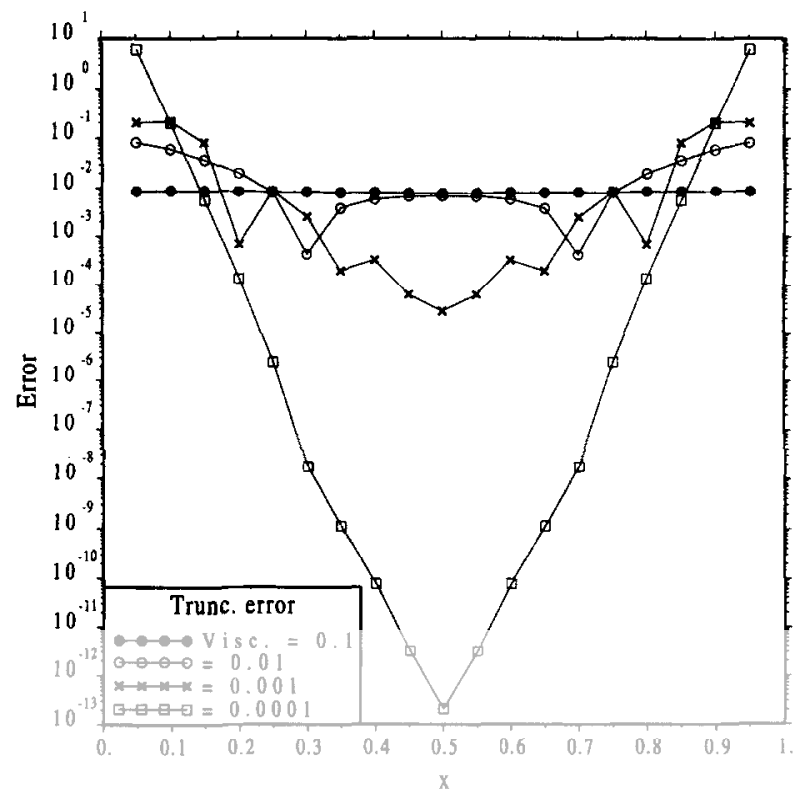

Fig. 1. Truncation error (multiplied by $h^{2} / \nu$ ) for the Galerkin solution of problem (14) using linear finite elements.

3. Galerkin/least-squares formulation

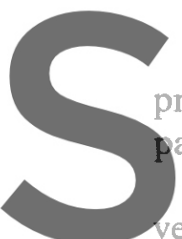

The methods to be described in what follows are motivated by

properties of the standard $G$

articular, we aim to impr

Galerkin approach

The first formulation tha

we discus

uss is the so-called Galerkin/least-squares

its actual formulation for advective-diffusive equations was introduced in [14]. The original motivation

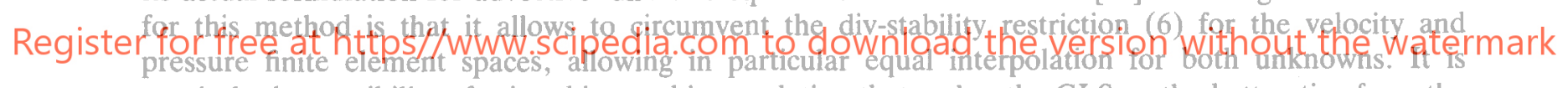

precisely the possibility of using this equal interpolation that makes the GLS method attractive from the computational standpoint. Here, we describe its extension to the probiem we are considering. For that, let us write Eqs. (1) as

$$
S(u, p)=\left(S_{1}(u, p), S_{2}(u, p)\right)=(f, 0)
$$

where we have introduced the vector operator $S$, with components

$$
\begin{aligned}
& S_{1}(u, p):=-\nu \Delta u+\nabla p+\omega \times u \\
& S_{2}(u, p):=\nabla \cdot u .
\end{aligned}
$$

Later on, we will also make use of the operator

$$
\boldsymbol{S}_{1}^{u}(\boldsymbol{u}, \boldsymbol{p}):=\nabla p+\boldsymbol{\omega} \times \boldsymbol{u},
$$

which is equal to $S_{1}(u, p)$ without the viscous term.

The idea of the GLS method is to add to the discrete Galerkin formulation a stabilizing term. This term is the $L^{2}$ product within each element of the operator $S$ applied to the test functions with the residual in the element multiplied by a matrix $\tau$, that is, $\tau\left[S\left(u_{h}, p_{h}\right)-(f, 0)\right]$.

Let us denote by $\left\{\Omega^{e}\right\}$ the finite element partition of the domain $\Omega$, with index $e$ ranging from 1 to the number of elements $n_{\mathrm{el}}$. Instead of Eq. (9), the problem to be solved now is to find $u_{h} \in V_{h}$ and $p_{h} \in Q_{h}$ such that

$$
\mathscr{A}\left(u_{h}, p_{h} ; v_{h}, q_{h}\right)-\mathscr{L}\left(v_{h}, q_{h}\right)+\mathscr{R}\left(u_{h}, p_{h} ; v_{h}, q_{h}\right)=0
$$


for all $\boldsymbol{v}_{h} \in V_{h}$ and $q_{h} \in Q_{h}$. If we take the matrix $\boldsymbol{\tau}$ as diagonal, with the terms corresponding to the momentum equations equal to a parameter $\tau_{1}$ and those corresponding to the continuity equation equal to $\tau_{2}$, the stabilizing term $\mathscr{R}\left(\boldsymbol{u}_{h}, p_{h} ; \boldsymbol{v}_{h}, q_{h}\right)$ is given by

$$
\mathscr{R}\left(\boldsymbol{u}_{h}, p_{h} ; \boldsymbol{v}_{h}, q_{h}\right)=\sum_{e=1}^{n_{\mathrm{el}}} \int_{\Omega^{e}}\left[\tau_{1} \boldsymbol{S}_{1}\left(\boldsymbol{v}_{h}, q_{h}\right) \cdot\left(\boldsymbol{S}_{1}\left(\boldsymbol{u}_{h}, p_{h}\right)-\boldsymbol{f}\right)+\tau_{2} S_{2}\left(\boldsymbol{v}_{h}, q_{h}\right) \cdot S_{2}\left(\boldsymbol{u}_{h}, p_{h}\right)\right] \mathrm{d} \Omega .
$$

The choice for the parameters $\tau_{1}$ and $\tau_{2}$ is discussed below.

In expanded form Eq. (30) leads to two equations, one corresponding to the approximation of Eq. (1a) and another to Eq. (1b). With the term $\mathscr{R}$ defined above, this expanded form reads

$$
\begin{aligned}
& l\left(\boldsymbol{v}_{\boldsymbol{h}}\right)= a\left(\boldsymbol{u}_{h}, \boldsymbol{v}_{h}\right)-\boldsymbol{b}\left(\boldsymbol{p}_{h}, \boldsymbol{v}_{h}\right)+c\left(\boldsymbol{u}_{h}, \boldsymbol{v}_{h}\right) \\
&+\sum_{e=1}^{n_{\mathrm{el}}} \int_{\Omega^{e}}\left[\tau_{1}\left(-\nu \Delta \boldsymbol{v}_{h}+\boldsymbol{\omega} \times \boldsymbol{v}_{h}\right) \cdot\left(-\nu \Delta \boldsymbol{u}_{h}+\nabla p_{h}+\boldsymbol{\omega} \times \boldsymbol{u}_{h}-\boldsymbol{f}\right)\right. \\
&\left.+\tau_{2}\left(\nabla \cdot w_{h}\right)\left(\nabla \cdot u_{h}\right)\right] \mathrm{d} \Omega \\
& 0=b\left(q_{h}, u_{h}\right)+\sum_{e=1}^{n_{\mathrm{e} 1}} \int_{\Omega^{e}} \tau_{1} \nabla q_{h} \cdot\left(-\nu \Delta u_{h}+\nabla p_{h}+\omega \times u_{h}-f\right) \mathrm{d} \Omega
\end{aligned}
$$

for all $v_{h} \in V_{h}$ and $q_{h} \in Q_{h}$

It is interesting at this point to write the matrix structure of the algebraic system resulting from problem (32), that is
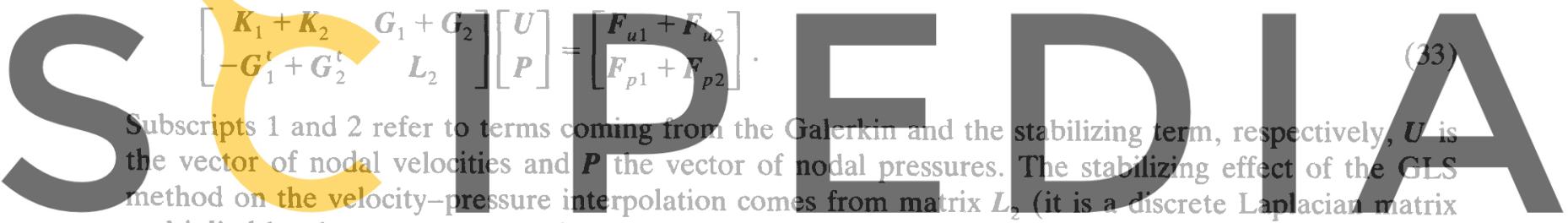

multiplied by the parameter $\tau_{1}$ within each element).

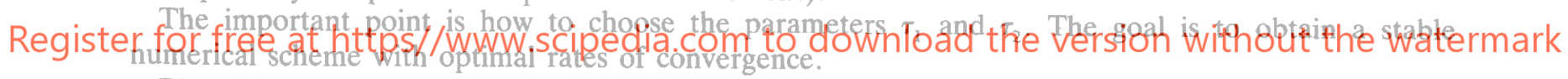

The bilinear form associated with problem (30) is

$$
\begin{aligned}
\mathscr{A}_{\mathrm{GLS}}\left(\boldsymbol{u}_{h}, p_{h} ; \boldsymbol{v}_{h}, q_{h}\right)= & \mathscr{A}\left(\boldsymbol{u}_{h}, p_{h} ; \boldsymbol{v}_{h}, q_{h}\right) \\
& +\sum_{\rho=1}^{n_{\mathrm{el}}} \int_{\Omega^{e}}\left[\tau_{1} S_{1}\left(\boldsymbol{v}_{h}, q_{h}\right) \cdot S_{1}\left(\boldsymbol{u}_{h}, p_{h}\right)+\tau_{2} S_{2}\left(\boldsymbol{v}_{h}, q_{h}\right) \cdot S_{2}\left(u_{h}, p_{h}\right)\right] \mathrm{d} \Omega .
\end{aligned}
$$

and therefore we have that

$$
\mathscr{A}_{\mathrm{GLS}}\left(\boldsymbol{u}_{h}, p_{h} ; \boldsymbol{u}_{h}, p_{h}\right)=\nu\left\|\nabla \boldsymbol{u}_{h}\right\|_{0}^{2}+\sum_{e=1}^{n_{\mathrm{el}}} \tau_{1}\left\|\boldsymbol{S}_{1}\left(\boldsymbol{u}_{h}, p_{h}\right)\right\|_{0, \Omega^{e}}^{2}+\sum_{e=1}^{n_{\mathrm{el}}} \tau_{2}\left\|S_{2}\left(\boldsymbol{u}_{h}, p_{h}\right)\right\|_{0, \Omega^{e}}^{2}
$$

where $\|\cdot\|_{0, \Omega^{e}}$ denotes the $L^{2}$ norm restricted to the eth element of the partition. In view of Eq. (35), we take the algorithm parameters $\tau_{1}$ and $\tau_{2}$ as

$$
\begin{array}{ll}
\tau_{1}=\frac{\beta h^{2}}{4 \nu}, & \text { with } \beta=\min \left\{C_{1 \beta} E k_{h}, C_{2 \beta}\right\} \\
\tau_{2}=\frac{\gamma \omega^{2} h^{4}}{\nu}, & \text { with } \gamma=\min \left\{C_{1 \gamma} E k_{h}, C_{2 \gamma}\right\},
\end{array}
$$

where $C_{1 \beta}, C_{2 \beta}, C_{1 \gamma}$ and $C_{2 \gamma}$ are constants that depend on the type of element being used. The parameters $\tau_{1}$ and $\tau_{2}$ are evaluated for each element, $h$ being its diameter.

When $\omega \rightarrow 0$ and $\nu$ is fixed we have that $\tau_{1} \rightarrow C_{2 \beta} h^{2} / 4 \nu$ and $\tau_{2} \rightarrow 0$, thus recovering the expressions used for example in [13], known to yield optimal rates of convergence for the problem without Coriolis 
force. In this particular case, the way we compute $C_{2 \beta}$ is based on the study of the GLS method applied to the one-dimensional convection-diffusion equation and considering the limiting situation of zero convection. In this case, the parameter $\tau_{1}$ can be computed as $\tau_{1}=\alpha h / 2|u|$, $u$ being now the convective velocity and $\alpha$ being a function of the Péclet Number $\mathrm{Pe}=|\boldsymbol{u}| h / 2 \nu$. For small values of this dimensionless number it is known that $\alpha$ must behave like $C_{2 \beta} \mathrm{Pe}$, with $C_{2 \beta}$ constant [14], thus leading precisely to expression (36a) (with $\mathrm{Ek}_{h}=0$ ) for $\tau_{1}$. it turns out that the constant $C_{2 \beta}$ can be taken as $1 / 3$ for linear elements and 1/9 for quadratics [15]. These are the values that we use in our calculations.

On the other hand, when $\nu \rightarrow 0$ from Eq. (35) and from the expression of the functions $\beta$ and $\gamma$ it is found that

$$
\mathscr{A}_{\mathrm{GLS}}\left(\boldsymbol{u}_{h}, p_{h} ; \boldsymbol{u}_{h}, p_{h}\right) \geqslant \sum_{e=1}^{n_{\mathrm{el}}} \frac{C_{1 \beta}}{4 \omega}\left\|\boldsymbol{\omega} \times \boldsymbol{u}_{h}+\nabla p_{h}\right\|_{0, \Omega^{e}}^{2}+\sum_{e=1}^{n_{\mathrm{el}}} C_{1 \gamma} \omega h^{2}\left\|\nabla \cdot \boldsymbol{u}_{h}\right\|_{0, \Omega^{e}}^{2},
$$

which is the stability estimate of the GLS method in the limit $\nu \rightarrow 0$. It is seen that this stability is given by the constants $C_{1 \beta}$ and $C_{1 \psi}$. The values for them that we have employed, as well as for the constant $C_{2 \gamma}$, are based on numerical experimentation. They are indicated in Section 5, where numerical results are shown demonstrating the effectiveness of the GLS method to remove the oscillations of the Galerkin method due to the dominating Coriolis force.

Before closing this section, let us remark that it is also necessary to take $\tau_{2}>0$ for the Navier-Stokes equations (see $[16,17]$ ). In this case, the viscous force does not compete with the Coriolis force, but with the convective one. However, there is also a certain relaxation of the incompressibility for very small viscosities, and $\tau_{2}>0$ must be selected to improve the numerical answers and also the convergence behavior of iterative schemes.
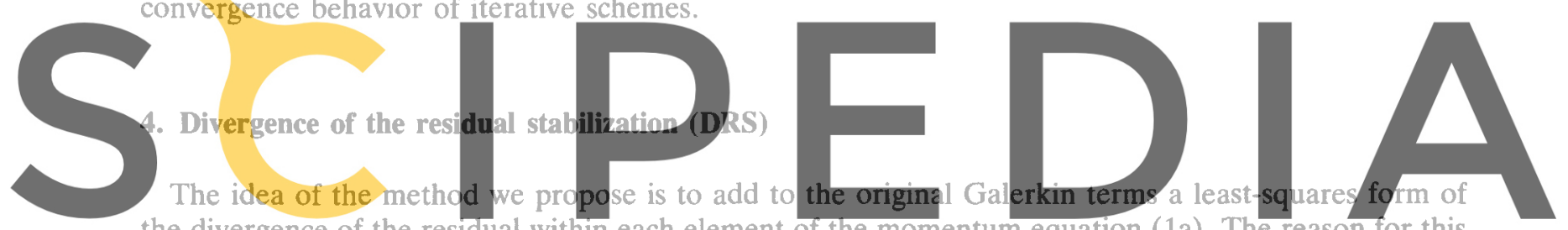

the divergence of the residual within each element of the momentum equation (1a). The reason for this is only the improvement of the stability that will be shown below. We call this approach divergence of

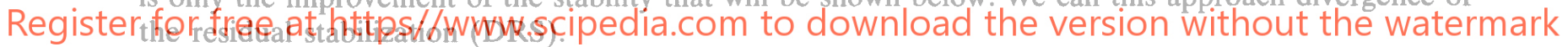

Instead of problem (30), we propose to solve the problem of finding $u_{h} \in V_{h}$ and $q_{h} \in Q_{h}$ such that

$$
\mathscr{A}\left(\boldsymbol{u}_{h}, p_{h} ; \boldsymbol{v}_{h}, q_{h}\right)-\mathscr{L}\left(\boldsymbol{v}_{h}, q_{h}\right)+\mathscr{D}\left(\boldsymbol{u}_{h}, p_{h} ; \boldsymbol{v}_{h}, q_{h}\right)=0
$$

for all $\boldsymbol{v}_{h} \in V_{h}$ and $q_{h} \in Q_{h}$.

The stabilization term $\mathscr{D}\left(u_{h}, p_{h} ; v_{h}, q_{h}\right)$ replaces the least squares form of the residual of the GLS formulation, that is, the term $\mathscr{R}\left(\boldsymbol{u}_{h}, p_{h} ; \boldsymbol{v}_{h}, q_{h}\right)$. Its expression is

$$
\mathscr{D}\left(\boldsymbol{u}_{h}, p_{h} ; \boldsymbol{v}_{h}, q_{h}\right)=\sum_{e=1}^{n_{\text {el }}} \int_{\Omega^{e}}\left[\tau \nabla \cdot S_{1}^{v}\left(\boldsymbol{v}_{h}, q_{h}\right)\left(\nabla \cdot S_{1}^{v}\left(u_{h}, p_{h}\right)-\nabla \cdot f\right)\right] \mathrm{d} \Omega
$$

and $\tau>0$ is a parameter that we take for each element as

$$
\tau=\delta \frac{h^{2}}{|\omega|}
$$

where $\delta$ is a function of the local Ekman number $\mathrm{Ek}_{h}$. A form for it dictated by a simple stability estimate is given below, although from numerical experiments we have observed that setting it equal to zero when $\mathrm{Ek}_{h}$ is high and taking it as a constant when it is small is an effective option. The particular values of this constant will be indicated in the next section.

The discrete variational equation (38) is consistent, in the sense that the exact solution of problem (1) satisfies it (for sufficiently smooth solutions). Observe that we have used the operator $S_{1}^{v}$ defined in Eq. (29) and not $S_{1}$, given by Eq. (28). Since the exact solution is divergence free, this keeps the consistency of the method, because 


$$
\nabla \cdot \boldsymbol{S}_{1}^{v}(u, p)=\nabla \cdot \boldsymbol{S}_{1}(u, p) .
$$

The use of $\boldsymbol{S}_{1}^{v}$ for the discrete problem avoids the need for computing the third derivatives of the shape functions, which would be clearly expensive and involved at the moment of implementing the method on the computer.

It is important to remark at this point that the added term $\mathscr{D}\left(\boldsymbol{u}_{h}, p_{h} ; \boldsymbol{v}_{h}, q_{h}\right)$ is not introduced to stabilize the velocity-pressure interpolation. As a consequence, we still need to use div-stable finite elements.

Let us consider now the expanded form of problem (38). It reads: find $\boldsymbol{u}_{h} \in V_{h}$ and $p_{h} \in Q_{h}$ such that

$$
\begin{aligned}
& l\left(\boldsymbol{v}_{h}\right)=a\left(\boldsymbol{u}_{h}, \boldsymbol{v}_{h}\right)-b\left(p_{h}, \boldsymbol{v}_{h}\right)+c\left(\boldsymbol{u}_{h}, \boldsymbol{v}_{h}\right) \\
& \quad+\sum_{e=1}^{n_{\text {el }}} \int_{\Omega^{e}} \tau[\nabla \cdot(\boldsymbol{\omega} \times \boldsymbol{v})]\left[\Delta p_{h}+\nabla \cdot\left(\boldsymbol{\omega} \times \boldsymbol{u}_{h}\right)-\nabla \cdot \boldsymbol{f}\right] \mathrm{d} \Omega \\
& 0=b\left(q_{h}, u_{h}\right)+\sum_{e=1}^{n_{e l}} \int_{\Omega^{e}} \tau \Delta q_{h}\left[\Delta p_{h}+\nabla \cdot\left(\omega \times u_{h}\right)-\nabla \cdot f\right] \mathrm{d} \Omega
\end{aligned}
$$

\section{for all $v \in V_{h}$ and $q_{h} \in Q_{h}$.}

The matrix form of the algebraic system resulting from problem (42) is the same as for the GLS formulation, i.e. the one given by Eq. (33). However, now the matrix $L_{2}$ in this equation results from the integral of the product of two Laplacians of pressure shape functions evaluated element by element.

Therefore, this matrix is zero if the pressure interpolation is linear.

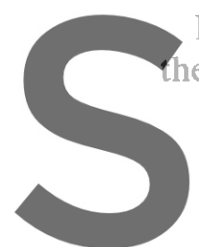

Let us consider precisely

he term $D$. The bilinear fo
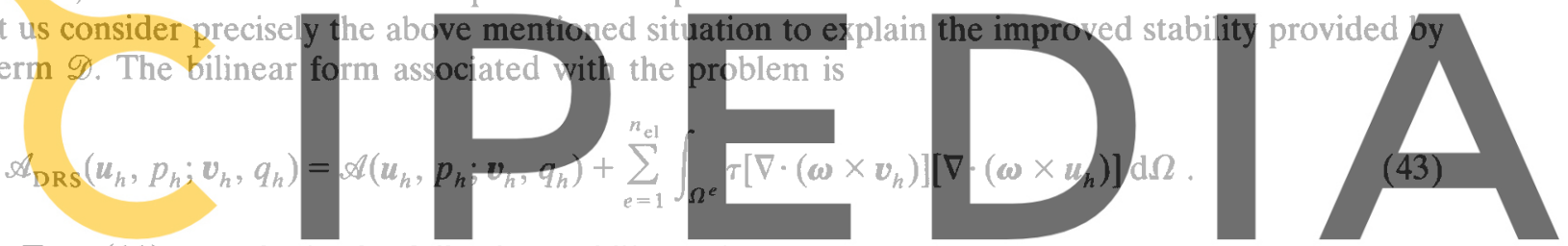

Using Eqs. (11), we obtain the following stability estimate

Register for free at https//www.scipedia.com to download the version without the watermark

$$
\mathscr{A}_{\mathrm{DRS}}\left(u_{h}, p_{h} ; u_{h}, q_{h}\right)=\nu\left\|\nabla u_{h}\right\|_{0}^{2}+\sum_{\rho=1} \tau\left\|\omega \cdot\left(\nabla \times u_{h}\right)\right\|_{0, \Omega^{e}}^{2} .
$$

Eq. (44) shows that we improve the stability of the original Galerkin formulation by gaining control over the term $\omega \cdot\left(\nabla \times u_{h}\right)$. If this term is zero (or very small), we retrieve the stability properties of problem (9). However, from Eq. (10) it is observed that the Galerkin method has good stability in this case and therefore therc is no need to modify it, except, perhaps, if one does not cven want to allow local oscillations near the boundary. In this sense, our motivation is completely different to that leading to the Galerkin-Gradient/least-squares formulation proposed in [8] for the scalar diffusion equation with a dominant absorption term, although apparently the idea is similar.

Taking into account the previous considerations, let us suppose that

$$
\left\|\boldsymbol{\omega} \cdot\left(\nabla \times \boldsymbol{u}_{h}\right)\right\|_{0, \Omega^{e}}^{2} \geqslant C_{\theta}|\boldsymbol{\omega}|^{2}\left\|\nabla \times \boldsymbol{u}_{h}\right\|_{0, \Omega^{e}}^{2}
$$

for a certain constant $C_{\theta}>0$ independent of the element $e$, and that the function $\delta$ in Eq. (40) is of the form

$$
\begin{aligned}
\boldsymbol{\delta}\left(\mathrm{Ek}_{h}\right) & =\min \left\{\frac{C_{1}}{\mathrm{Ek}_{h}}, C_{2}\right\} \\
& =\min \left\{C_{1} \frac{|\omega| h^{2}}{\nu}, C_{2}\right\},
\end{aligned}
$$

where $C_{1}$ and $C_{2}$ are positive constants. Assuming that in Eq. (43) $\tau$ is the same for all the elements, from Eq. (44) it follows that 


$$
\mathscr{A}_{\mathrm{DRS}}\left(\boldsymbol{u}_{h}, p_{h} ; \boldsymbol{u}_{h}, p_{h}\right) \geqslant \nu\left\|\nabla \boldsymbol{u}_{h}\right\|_{0}^{2}+\frac{C_{1} C_{\theta}}{\nu}|\boldsymbol{\omega}|^{2} h^{4}\left\|\nabla \times \boldsymbol{u}_{h}\right\|_{0}^{2}
$$

when $\mathrm{Ek}_{h}$ is high, that is, when the viscous force dominates, and

$$
\mathscr{A}_{\mathrm{DRS}}\left(\boldsymbol{u}_{h}, p_{h} ; \boldsymbol{u}_{h}, p_{h}\right) \geqslant \nu\left\|\nabla \boldsymbol{u}_{h}\right\|_{0}^{2}+C_{2} C_{\theta}|\boldsymbol{\omega}| h^{2}\left\|\nabla \times \boldsymbol{u}_{h}\right\|_{0}^{2}
$$

when the viscosity is small and therefore it is the Coriolis force the dominating one.

From the estimates (47) and (48) it is observed that the term added to the original Galerkin formulation has an important influence only when the viscosity is small, provided Eq. (45) holds true. In particular, from estimate (48) we see that the term on which we gain control is the curl of the velocity, i.e. the vorticity. On the other hand, the velocity is weakly solenoidal and is zero on the boundary. Under these conditions, a bound for the vorticity implies a bound for the whole velocity gradient $\nabla u_{h}$ (cf. [18]), which in turn results in an estimate for the velocity itself by using the Poincaré-Friedrics incquality. In conclusion, the stability is cnhanced.

\section{Numerical examples}

In this section we present the numerical results obtained using the formulations proposed in this paper for a simple 2D example. The domain is the sector of a centrifugal fan comprised between two flat blades. The angle between the axes of these blades is $45^{\circ}$, whereas they form an angle of $30^{\circ}$ with the cylinder of radius 1 to which they are fixed and they occupy a sector of $7.5^{\circ}$. The flow is confined by an outer cylinder of radius 2 , forming a gap of 0.2 with the blades. This is at rest and the fan is rotating anticlockwise at an angular velocity of $1 \mathrm{rad} / \mathrm{s}$, i.e. $\omega=2 \mathrm{rad} / \mathrm{s}$, so that the relative velocity of the outer cylinder has norm $|\boldsymbol{u}|=2$ and is tangent to it.

The boundary conditions for the velocity expressed in the reference system fixed to the rotating fan are zero velocity on the blades and the inner cylinder, prescribed tangent velocity on the outer one, tangent velocity on the upper part of the blades (concentric with the cylinders) and periodic velocity on the inlet and outlet of the domain.

The computational domain and a finite element mesh of bilinear elements is shown in Fig. 2(1). This mesh consists of 461 nodal points and 416 elements. We shall refer to it as 'mesh 1'. Also, two other finite element meshes will be considered, obtained by splitting successively each element into four. They will be referred to as 'mesh 2' and 'mesh 3', respectively. Their number of nodal points is 1753 for

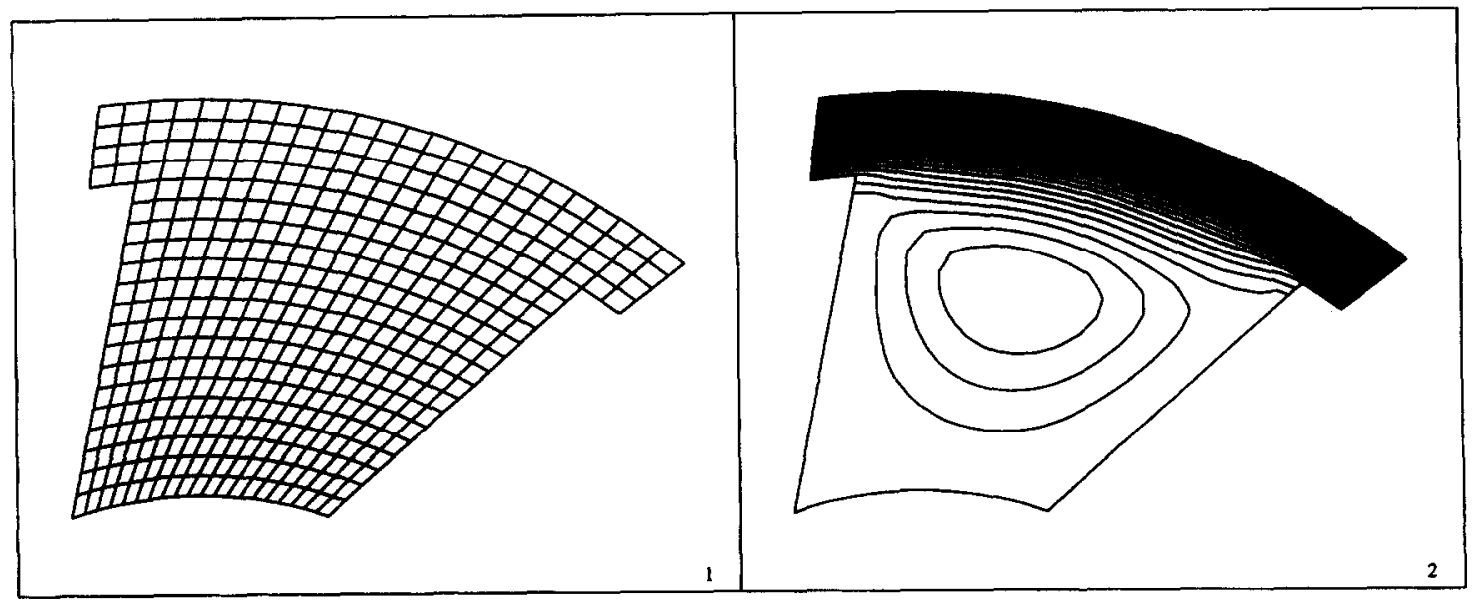

Fig. 2. (1) Computational domain and mesh 1 in the case of bilinear elements (461 nodal points). (2) Streamlines for the case $\omega=0$ and using the $Q_{2} / Q_{2}$ element. The peaks of the streamfunction are 0.342 and -0.0479 . 
mesh 2 and 6833 for mesh 3. Also, we shall consider the use of biquadratic elements whose finite element meshes are obtained by grouping together four bilinear elements of meshes 1,2 and 3 .

We shall solve numerically Eqs. (1) with a value of the viscosity $\nu=5 \times 10^{-8}$, for which oscillatory results are obtained using the standard Galerkin method.

The only body force that we shall consider is the centrifugal one, given by $f=1 / 4 \omega \times(\omega \times r)$, where $\boldsymbol{r}$ is the vector of position of the particles. If the viscous term is small, the pressure gradient will be approximately equal to $f$.

The exact solution to this problem is exactly the same as that of the Stokes problem without Coriolis force. This is due to that fact that for $2 \mathrm{D}$ incompressible flows the Coriolis force is curl-free, since

$$
\nabla \times(\omega \times u)=\omega \nabla \cdot u=0 .
$$

Thus, the Coriolis force must be the gradient of a scalar function that can be included in the pressure. Of course, this can not be done if the tension is prescribed on part of the boundary, since in this case we have a condition involving the physical pressure.

The streamline pattern obtained with $\omega=0$ and using the $Q_{2} / Q_{1}$ element (continuous biquadratic velocity, continuous bilinear pressure), known to satisfy the div-stability condition (6) [18], is shown in Fig. 2(2). The coarsest mesh (mesh 1) has been used. This result will serve us as a reference for the following cases with $\omega=2$.

Let us consider first the use of bilinear elements. It is well known that there is no mixed velocity-pressure interpolation with continuous bilinear velocities satisfying condition (6). However, the $Q_{1} / P_{0}$ element, with piecewise constant pressures, is known to yield good results most of the times, even though a spurious pressure mode needs to be filtered in some cases (see [1] for further discussion about this controversial element). We have used it in this example and, as we shall see, with good results.

The streamlines obtained using the Galerkin method with mesh 1 of $Q_{1} / P_{0}$ elements are shown in Fig. 3(1). It is observed that the solution is completely oscillatory, as well as the pressure contours shown in Fig. 3(2). The solution improves as the mesh is refined, although in this case the streamline pattern is still very bad using mesh 3 (Fig. 3(3)). Pressures are not so bad using this mesh, mainly because the component due to the centrifugal force dominates (Fig. 3(4)).

As a first alternative to stabilize the Galerkin formulation, let us consider the use of the GLS method. For this problem, the local Ekman number is $\mathrm{Ek}_{h}=2.5 \times 10^{-8} h^{-2}$, where $h$ is the size of the element for which $\mathrm{Ek}_{h}$ is computed. For this value of $\mathrm{Ek}_{h}$ and for the values of the constants appearing in Eq. (36) that we use and that are indicated below, the expressions for the parameters $\tau_{1}$ and $\tau_{2}$ reduce to

$$
\tau_{1}=\frac{C_{1 \beta}}{4 \omega}, \quad \tau_{2}=C_{1 \gamma} \omega h^{2}
$$

In order to determine the best values for the constants $C_{1 \beta}$ and $C_{1 \gamma}$ we have sought numerically the values that give the closest results to those shown in Fig. 2(2). After a series of numerical experiments we have found that good solutions are obtained with

$$
C_{1 \beta}=4, \quad C_{1 \gamma}=10 \quad\left(Q_{1} \text { element }\right)
$$

for the whole set of meshes 1,2 and 3. All the numerical results using stabilized methods shown in what follows have been obtained using mesh 1 . In Fig. 4(1) we have plotted the streamlines that are obtained using the GLS method with $\tau_{2}=0$ and computing $\tau_{1}$ as in the case without Coriolis force, that is, using the expression given in Eq. (36a) but with $\beta=C_{2 \beta}=1 / 3$, a constant instead of a function of the Ekman number. It is clearly seen that the solution is bad, although no oscillations are present. In particular, the incompressibility has been poorly approximated, and our algorithm to compute the streamfunction from the velocity field, which is based on the fact that the solution is divergence-free, yields streamlines with the origin on the blades. Figs. $4(2)$ and $4(3)$ show the results obtained first with $C_{1 \gamma}=0$ and with $C_{1 \beta}=4$ and then with this value of $C_{1 \beta}$ and $C_{1 \gamma}=10$. It can be observed that in this last case there is a better approximation of the incompressibility constraint (although the combination given in Eq. (50) is the best we have found, if $C_{1 \gamma}=0$ there are values of $C_{1 \beta}$ that give better results than 4). To complete 


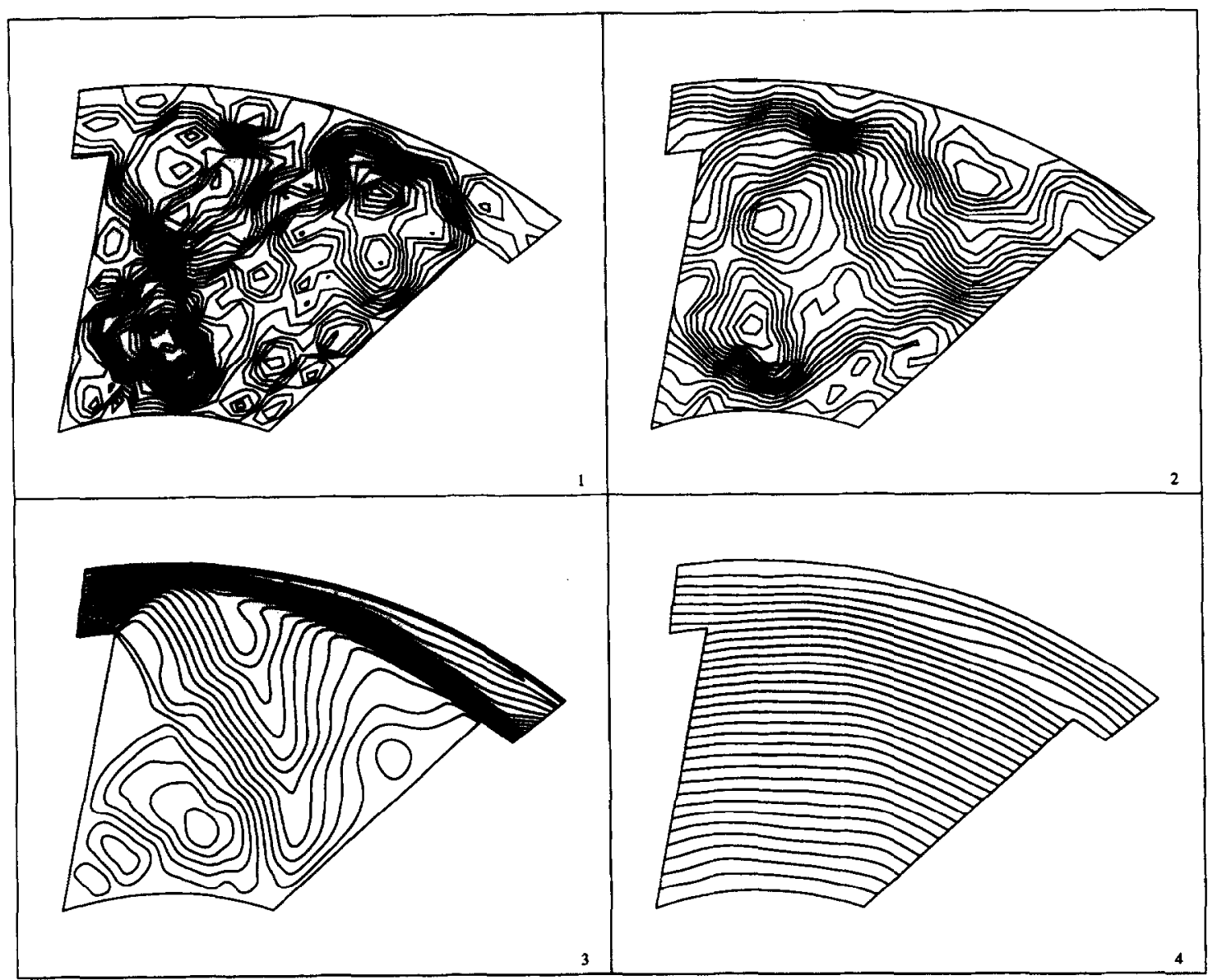

Fig. 3. Solution using the Galerkin method with the $Q_{1} / P_{0}$ element. (1) Streamlines. Mesh 1. (2) Pressure contours. Mesh 1. (3) Streamlines. Mesh 3. (4) Pressure contours. Mesh 3.

the definition of the functions $\beta$ and $\gamma$ in Eq. (36), we take $C_{2 \beta}=1 / 3$, as already mentioned, and $C_{2 \gamma}=5 / 6$, so that $\gamma=5 \beta / 2$.

The solution obtained using the $Q_{1} / P_{0}$ element and the DRS technique is shown in Fig. 4(4). We have taken $\delta=0.02$ in this case (see Eq. (40)). It is observed that the solution is better than using the GLS method, with a better approximation of the incompressibility and with peaks of the streamfunction closer to those obtained in the case $w=0$ (Fig. 2(2)).

In order to compare the quality of the numerical results, we have plotted in Fig. 5 the velocity variation along the straight line joining the centers of the sectors of the two concentrical cylinders that enclose the computational domain. Hereafter, we refer to this line as the 'middle section'. Taking as a reference the curve corresponding to the case $\omega=0$ obtained with the $Q_{1} / P_{0}$ element and the Galerkin approach, it is observed that the results using the DRS method are slightly better than using the GLS technique. In Fig. 5 we have also plotted the solution obtained using the Galerkin method and mesh 3 (the finest one) to see the type of oscillations encountered. The pressure variation along the middle section is shown in Fig. 6. It is observed that the solution obtained using the Galerkin method on mesh 3 is basically that due to the centrifugal force, without reproducing the pressure decrease close to the outer cylinder that can be observed using the GLS or the DRS methods.

Let us consider now the case of biquadratic elements. The streamlines obtained using the Galerkin method on meshes 1, 2 and 3 are shown in Figs. 7(1), 7(2) and 7(3), respectively. It is clearly observed how the solution improves as the mesh is refined. Using mesh 3 there are no oscillations in the 


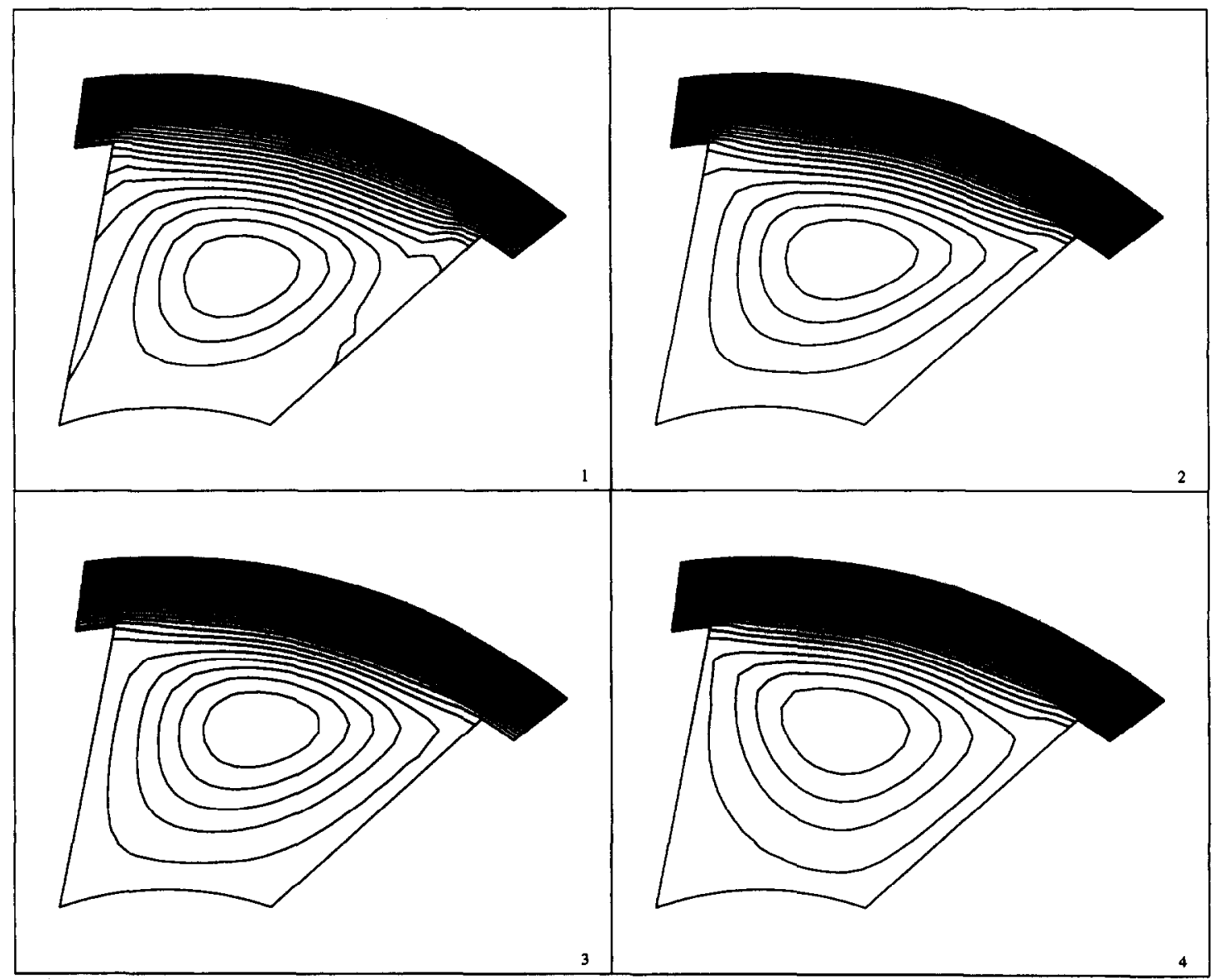

Fig. 4. Streamlines using stabilized methods with $Q_{1}$ elements. (1) GLS method, $\beta=1 / 3, \gamma=0$. Streamfunction peaks: 0.444 and -0.0637 . (2) GLS method, $C_{1 \beta}=4, C_{1 \gamma}=0$. Streamfunction peaks: 0.361 and -0.0613 . (3) GLS method, $C_{1 \beta}=4, C_{1 \gamma}=10$. Streamfunction peaks: 0.322 and -0.0672 . (4) DRS method $\left(Q_{1} / P_{0}\right.$ element $), \delta=0.02$. Streamfunction peaks: 0.346 and -0.0546 .

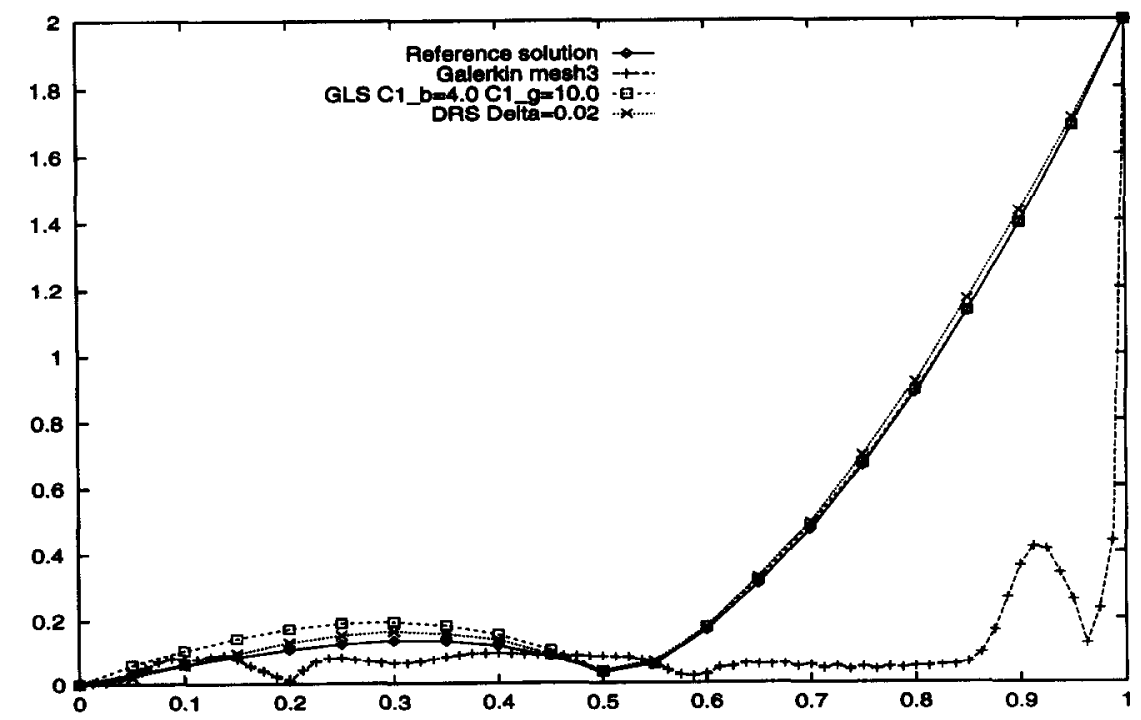

Fig. 5. Velocity variation along the middle section using $Q_{1}$ elements $\left(C 1_{-} b \equiv C_{1 \beta}\right.$ and $\left.C 1_{-} g \equiv C_{1 \gamma}\right)$. The reference solution is computed with $\omega=0$ and the $Q_{1} / P_{0}$ element. 


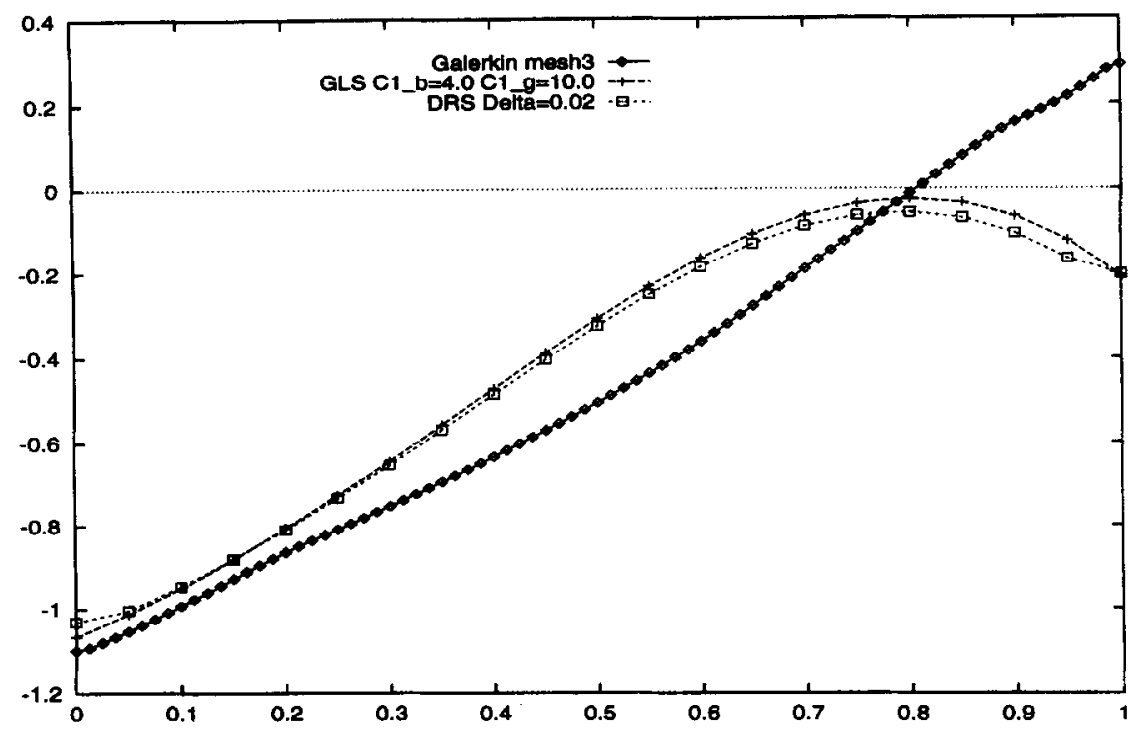

Fig. 6. Pressure variation along the middle section using $Q_{1}$ elements $\left(C 1_{-} b \equiv C_{1 \beta}\right.$ and $\left.C 1_{-} g \equiv C_{1 \gamma}\right)$.

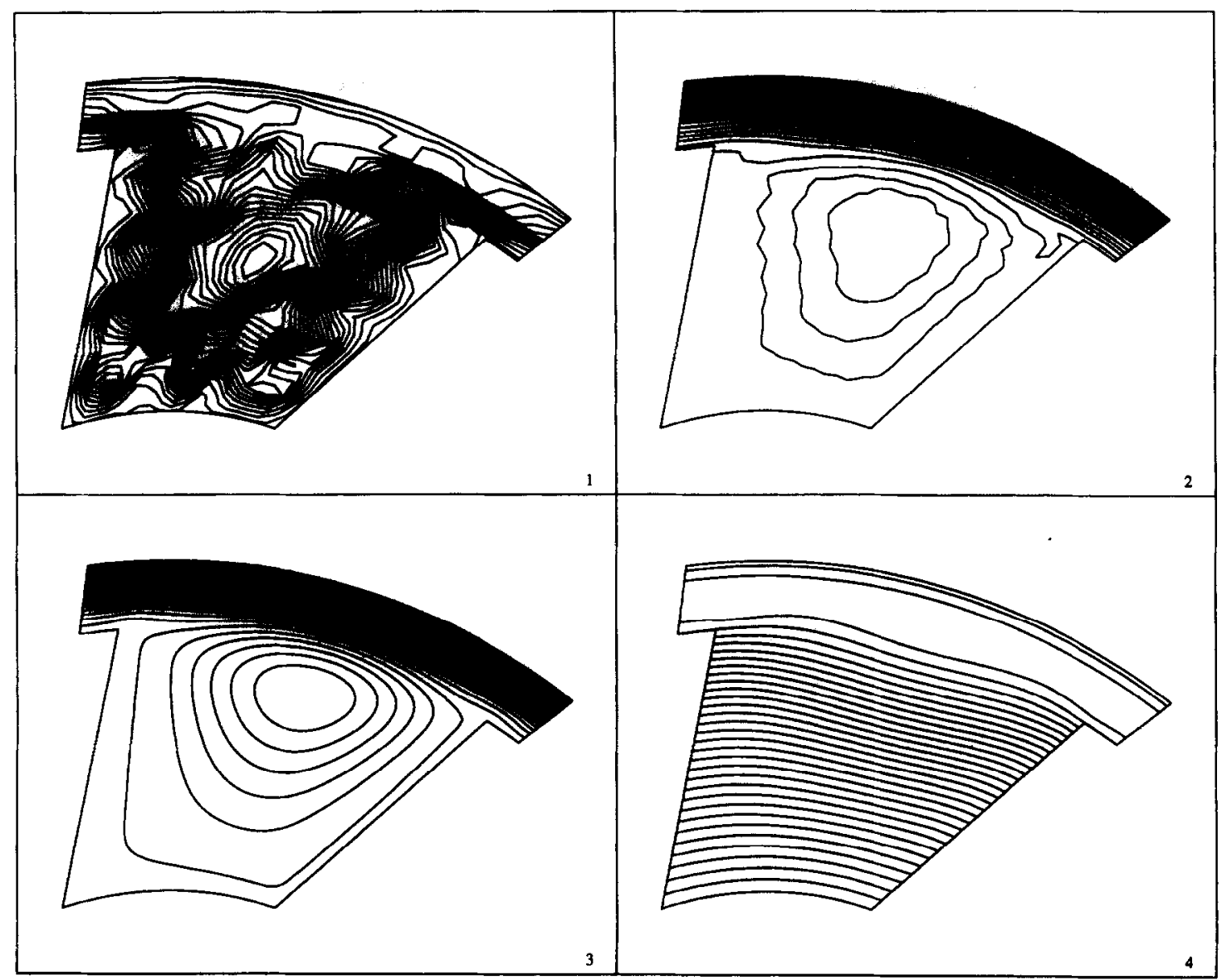

Fig. 7. Solution using the Galerkin method with the $Q_{2} / Q_{1}$ element, (1) Streamlines. Mesh 1. (2) Streamlines. Mesh 2. (3) Streamlines. Mesh 3. Streamfunction peaks: 0.203 and -0.0502 . (4) Pressure contours. Mesh 3. 
streamlines, although the position of the central vortex is still not correct if we compare it with the result obtained in Fig. 2(2). Also, the streamfunction peaks have an important error. Pressure contours using mesh 3 are plotted in Fig. 7(4).

Fig. 8(1) shows the streamlines obtained using equal velocity-pressure interpolation and the GLS method with $\beta=1 / 9$ and $\gamma=0$, which are the parameters that yield the best results in the case $\omega=0$. Clearly, this solution is wrong. The incompressibility condition is far from being approximated and the fluid flows basically close to the outer cylinder. Using the same strategy as for the $Q_{1}$ element, we have found that good results are obtained if Eq. (49) is used with

$$
C_{1 \beta}=0.4, \quad C_{1 \gamma}=1 \quad\left(Q_{2} \text { element }\right) .
$$

The solution obtained with this value of $C_{1 \beta}$ and $C_{1 \gamma}=0$ is shown in Fig. 8(2), whereas the solution with $C_{1 \gamma}=1$ is shown is Fig. 8(3). Pressure contours obtained in this last case are shown in Fig. 8(4). For this element, we use the values of the constants in Eq. (51) and $C_{2 \beta}=1 / 9$ and $C_{2 \gamma}=5 / 18$ (and thus $\gamma=5 \beta / 2$, as for the $Q_{1}$ element).

Concerning the use of the DRS method, we have tested both the $Q_{2} / Q_{1}$ and the $Q_{2} / P_{1}$ (continuous biquadratic velocity, piecewise linear pressure) elements. The streamline pattern obtained in both cases is plotted in Figs. 9(1) and $9(2)$, showing a very good agreement with the reference solution of Fig. 2(2). For the $Q_{2} / Q_{1}$ element we have taken $\delta=0.001$ and for the $Q_{2} / P_{1} \delta=0.02$.

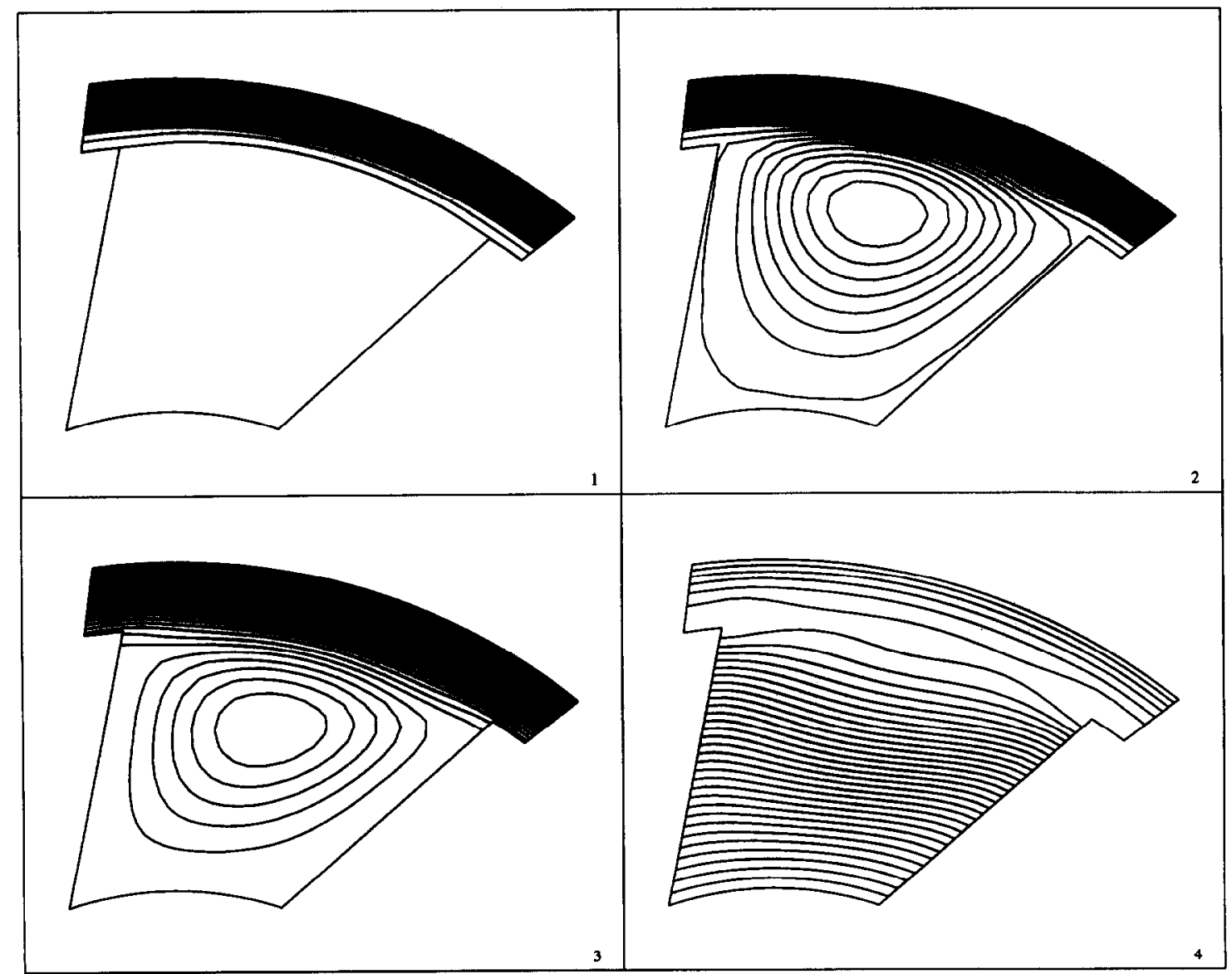

Fig. 8. Results using the GLS method with $Q_{2}$ elements. (1) Streamlines, $\beta=1 / 9, \gamma=0$. Streamfunction peaks: 0.182 and -0.00583 . (2) Streamlines, $C_{1 \beta}=0.4, C_{1 \gamma}=0$. Streamfunction peaks: 0.225 and -0.0791 . (3) Streamlines, $C_{1 \beta}=0.4, C_{1 \gamma}=1$. Streamfunction peaks: 0.310 and -0.0699 . (4) Pressure contours. 


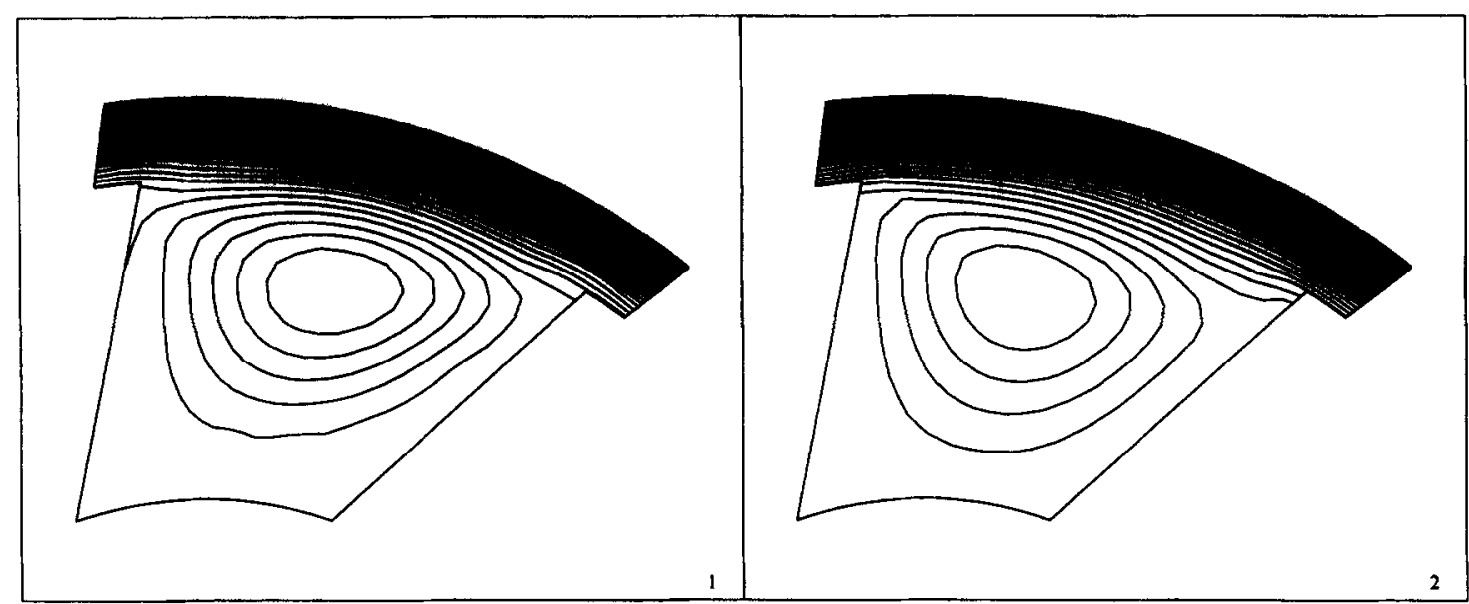

Fig. 9. Streamlines using the DRS method with $Q_{2}$ elements. (1) $Q_{2} / Q_{1}$ elements, $\delta=0.001$. Streamfunction peaks: 0.302 and -0.0735 . (2) $Q_{2} / P_{1}$ elements, $\delta=0.02$. Streamfunction peaks: 0.344 and -0.0667 .

Figs. 10 and 11 show respectively the velocity and pressure variation along the middle section using the Galerkin method on mesh 3, the DRS method using $Q_{2} / Q_{1}$ and $Q_{2} / P_{1}$ elements and the GLS method using biquadratic velocity-pressure interpolation.

Finally, to see the performance of the stabilization techniques discussed herein on unstructured meshes we have solved the problem using the mesh of 441 triangles shown in Fig. 12(1). Streamlines obtained using the GLS method and the mesh of $P_{1}$ elements obtained by splitting each triangle into four are shown in Fig. 12(2) (the total number of nodal points is 968), whereas results obtained using $P_{2}$ elements are plotted in Fig. 12(3). For the $P_{1}$ element we have used the values of the constants given in Eq. (50) and for the $P_{2}$ we have taken $C_{1 \beta}=0.004$ and $C_{1 \gamma}=1$. For the DRS method we have used the $P_{2}^{+} / P_{1}$ element (continuous quadratic velocities enriched with bubble functions and discontinuous piecewise linear pressures), eliminating the degrees of freedom corresponding to the internal bubbles using static condensation. The streamlines obtained in this case are shown in Fig. 12(4). These results have been computed with $\delta=0.02$.

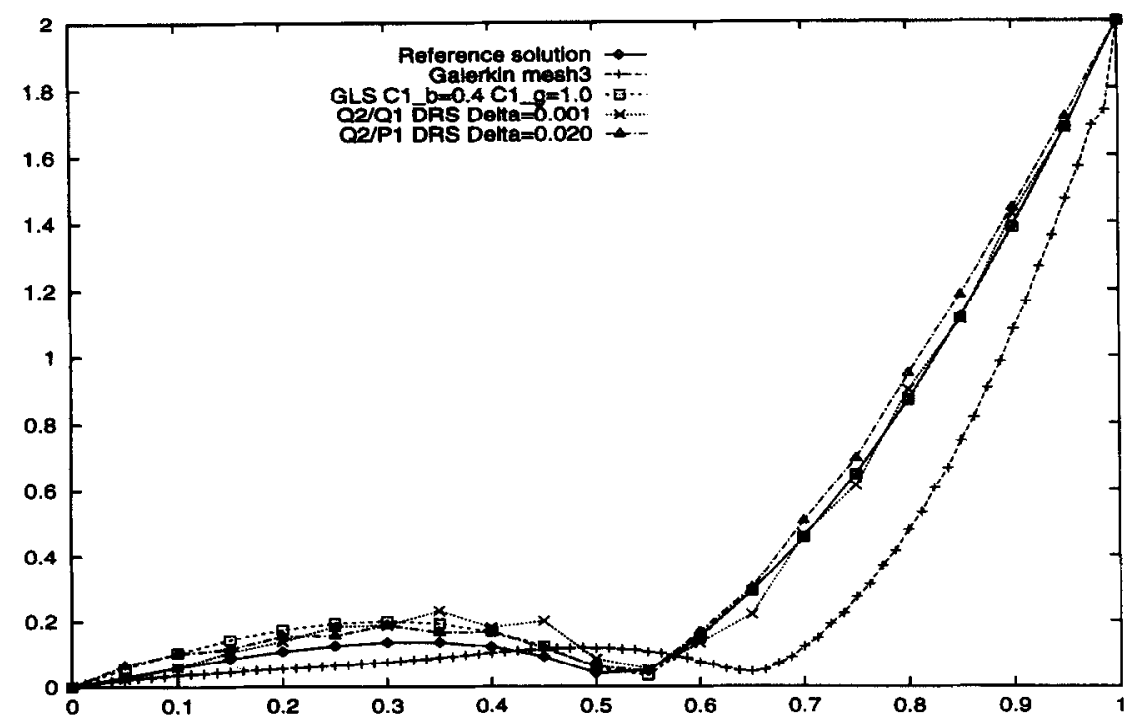

Fig. 10. Velocity variation along the middle section using $Q_{2}$ elements $\left(C 1_{-} b \equiv C_{1 \beta}\right.$ and $\left.C 1_{-} g \equiv C_{1 \gamma}\right)$. The reference solution is computed with $\omega=0$ and the $Q_{2} / Q_{1}$ element. 


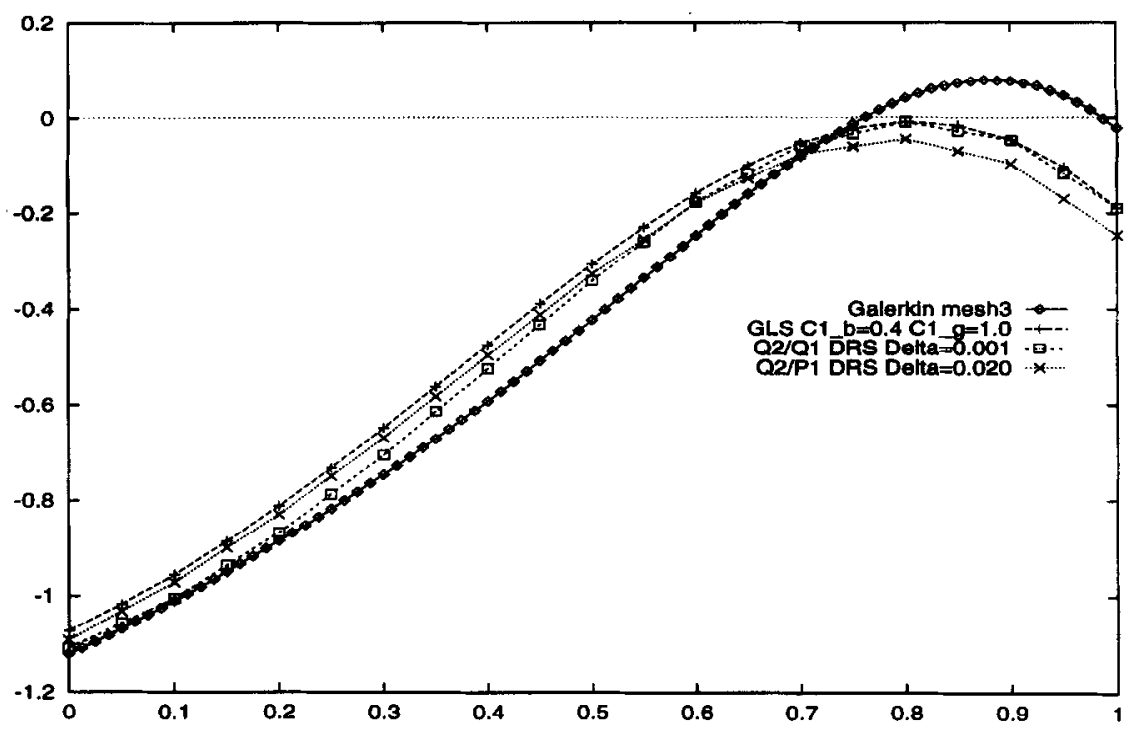

Fig. 11. Pressure variation along the middle section using $Q_{2}$ elements $\left(C 1_{-} b \equiv C_{1 \beta}\right.$ and $\left.C 1_{-} g \equiv C_{1 \gamma}\right)$.

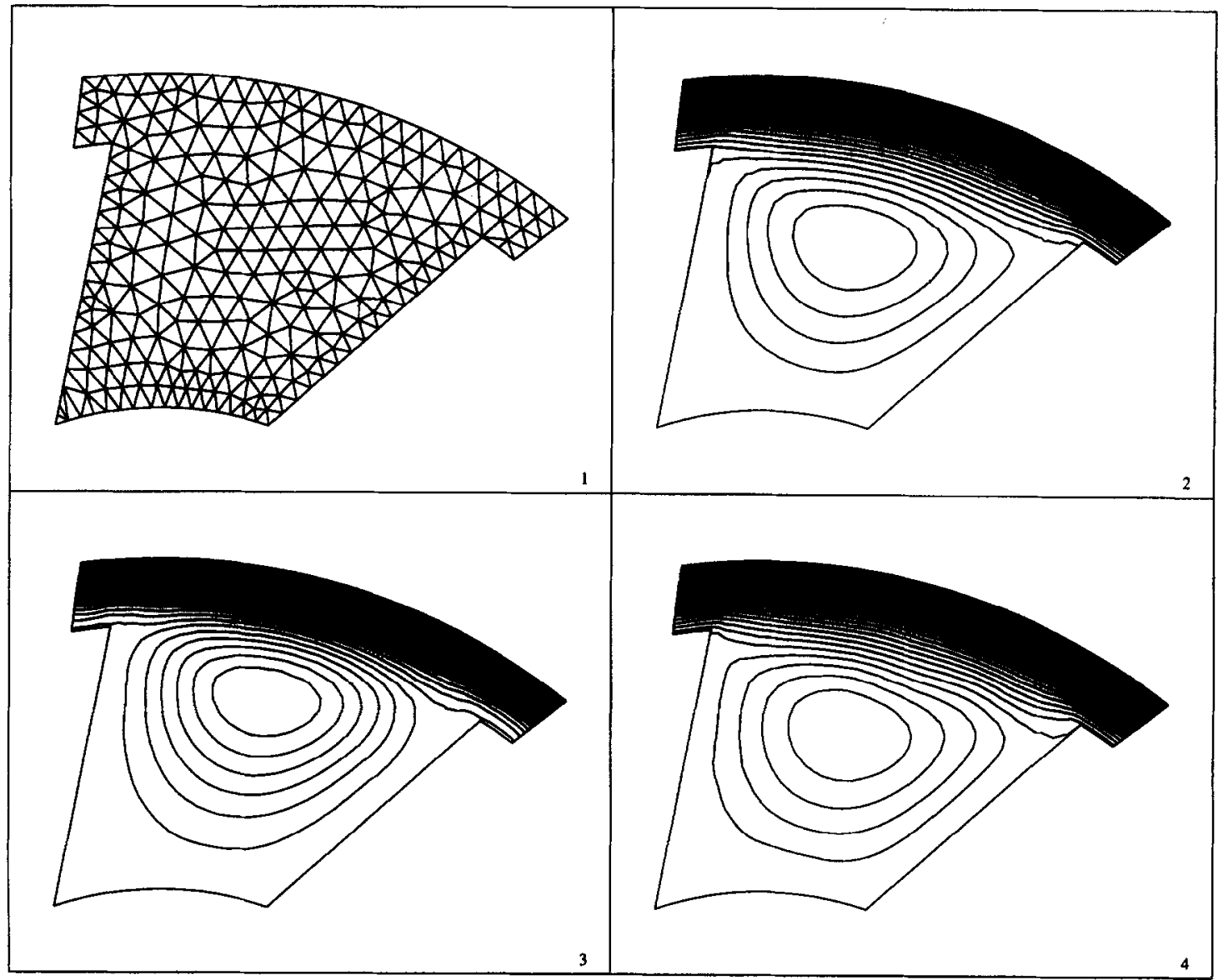

Fig. 12. (1) Unstructured mesh of triangles. (2) Streamlines using the GLS method with $P_{1}$ elements. $C_{1 \beta}=1, C_{1 \gamma}=10$. Streamfunction peaks: 0.354 and -0.00151 . (3) Streamlines using the GLS method with $P_{2}$ elements. $C_{1 \beta}=0.001, C_{1 \gamma}=1$. Streamfunction peaks: 0.318 and -0.00534 . (4) Streamlines using the DRS method with $P_{2}^{+} / P_{1}$ elements. $\delta=0.02$. Streamfunction peaks: 0.372 and -0.000347 . 


\section{Conclusions}

In this paper we have discussed the problems encountered when one considers the presence of a dominating Coriolis force in the Stokes equations. We have shown that oscillations occur when the standard Galerkin formulation is used. To overcome this misbehavior, two different possibilities have been studied. The first of them is the GLS method, for which we have proposed expressions for computing the numerical parameters that define this method. The second formulation is novel, and based on the addition of a least-squares form of the divergence of the residual of the momentum equations to the basic Galerkin terms. We have given theoretical indications to explain why the stability is enhanced. From the numerical experiments that we have carried out it is observed that the performance of this method is excellent. It precludes the numerical oscillations without being excessively overdiffusive.

Concerning the extension of this technique to the incompressible Navier-Stokes equations, it has to be pointed out that the values of the Ekman number for which the Galerkin formulation fails correspond to extremely high values of the Reynolds number. Therefore, in realistic physical situations the problem of important Coriolis force appears together with complicated flow behavior and, probably, turbulence. We think that the model problem studied in this paper and the techniques designed to solve it will allow to discern the sources of numerical difficulties.

\section{Acknowledgment}

This work has been partially supported by BRITE-EURAM Project Number 5076, entitled Development of a Computer-aided Methodology for Enhanced Design of Axial and Centrifugal Fans. This support is gratefully acknowledged.

\section{References}

[1] F. Brezzi and M. Fortin, Mixed and Hybrid Finite Element Methods (Springer-Verlag, 1991).

[2] F. Brezzi and J. Douglas, Stabilized mixed methods for the Stokes problem, Numer. Math. 53 (1988) 225-235.

[3] I. Franca and T.J.R. Hughes, Two classes of mixed finite element methods, Comput. Methods Appl. Mech. Engrg. 69 (1988) 89-129.

[4] J. Douglas and J. Wang, An absolutely stabilized finite element method for the Stokes problem, Math. Comput. 52 (1989) 495-508.

[5] T.J.R. Hughes, The Finite Element Method. Linear Static and Dynamic Analysis (Prentice-Hall, Englewood Cliffs, NJ, 1987).

[6] O.C. Zienkiewicz and R.L. Taylor, The Finite Element Method, 4th edition, Vol. 1 (McGraw-Hill, 1989).

[7] D.J. Tritton, Physical Fluid Dynamics (Van Nostrand Reinhold, 1977).

[8] L. Franca and E.G. Dutra do Carmo, The Galerkin Gradient least-squares method, Comput. Methods Appl. Mech. Engrg. 74 (1989) 41-54.

[9] T.E. Tezduyar and Y.J. Park, Discontinuity-capturing finite element formulations for nonlinear convection-diffusionreaction equations, Comput. Methods Appl. Mech. Engrg. 59 (1986) 307-325.

[10] R. Codina, A shock-capturing anisotropic diffusion for the finite element solution of the diffusion-convection-reaction equation, in: K. Morgan, E. Oñate, J. Periaux, J. Peraire and O.C. Zienkiewicz, eds., Finite Elements in Fluids VIII, Part I (CIMNE/Pineridge Press, 1993) 67-75.

[11] T.J.R. Hughes, L.P. Franca and M. Balestra, A new finite element formulation for computational fluid dynamics: V. Circumventing the Babuška-Brezzi condition: a stable Petrov-Galerkin formulation for the Stokes problem accommodating equal-order interpolations, Comput. Methods Appl. Mech. Engrg. 59 (1986) 85-99.

[12] T.J.R. Hughes and L.P. Franca, A new finite element formulation for computational fluid dynamics: VII. The Stokes problem with various well-posed boundary conditions: symmetric formulations that converge for all velocity/pressure spaces, Comput. Methods Appl. Mech. Engrg. 65 (1987) 85-96.

[13] L. Franca and R. Stenberg, Error analysis of some Galerkin least-squares methods for the elasticity equations, SIAM J. Numer. Anal. 28 (1991) 1680-1697.

[14] T.J.R. Hughes, L.P. Franca and G.M. Hulbert, A new finite element formulation for computational fluid dynamics: VIII. The Galerkin/least-squares method for advective-diffusive equations, Comput. Methods Appl. Mech. Engrg. 73 (1989) 173-189. 
[15] R. Codina, E. Oñate and M. Cervera, The intrinsic time for the SUPG formulation using quadratic elements, Comput. Methods Appl. Mech. Engrg. 94 (1992) 239-262.

[16] L. Franca and S.L. Frey, Stabilized finite element methods: II. The incompressible Navier-Stokes equations, Comput. Methods Appl. Mech. Engrg. 99 (1992) 209-233.

[17] T.E. Tezduyar, S. Mittal, S.E. Ray and R. Shih, Incompressible flow computations with stabilized bilinear and linear equal-order-interpolation velocity-pressure elements, Comput. Methods Appl. Mech. Engrg. 95 (1992) $221-242$.

[18] V. Girault and P.A. Raviart, Finite Element Methods for Navier-Stokes Equations (Springer-Verlag, 1986). 